\title{
Design considerations for liquid crystal contact lenses
}

DOI:

10.1088/1361-6463/aa9358

\section{Document Version}

Accepted author manuscript

Link to publication record in Manchester Research Explorer

\section{Citation for published version (APA):}

Bailey, J., Kaur, S., Morgan, P. B., Gleeson, H. F., Clamp, J. H., \& Jones, J. C. (2017). Design considerations for liquid crystal contact lenses. Journal of Physics D: Applied Physics, 50(48), [485401]. https://doi.org/10.1088/13616463/aa9358

\section{Published in:}

Journal of Physics D: Applied Physics

\section{Citing this paper}

Please note that where the full-text provided on Manchester Research Explorer is the Author Accepted Manuscript or Proof version this may differ from the final Published version. If citing, it is advised that you check and use the publisher's definitive version.

\section{General rights}

Copyright and moral rights for the publications made accessible in the Research Explorer are retained by the authors and/or other copyright owners and it is a condition of accessing publications that users recognise and abide by the legal requirements associated with these rights.

\section{Takedown policy}

If you believe that this document breaches copyright please refer to the University of Manchester's Takedown Procedures [http://man.ac.uk/04Y6Bo] or contact uml.scholarlycommunications@manchester.ac.uk providing relevant details, so we can investigate your claim.

\section{OPEN ACCESS}


Design Considerations for Liquid Crystal Contact lenses.

J. Bailey ${ }^{1}$, S. Kaur ${ }^{1,2}$, P.B. Morgan ${ }^{3}$, H.F. Gleeson ${ }^{1}$, J.H. Clamp ${ }^{4}$ and J.C. Jones ${ }^{1, *}$

1. Soft Matter Physics, School of Physics and Astronomy, E.C. Stoner Building, University of Leeds, Leeds, LS2 9JT,

UK.

2. Merck Chemicals Ltd., University Parkway, Chilworth Science Park, SO16 7QD, UK.

3. Eurolens Research, University of Manchester, Carys Bannister Building, Dover Street, Manchester, UK, M13 9PL

4. UltraVision CLPL, Commerce Way, Leighton Buzzard, Bedfordshire, LU7 4RW, UK

*J.C.Jones@leeds.ac.uk

\begin{abstract}
Switchable liquid crystal contact lenses with electrically controllable focal powers have previously been investigated as an alternative to bifocal contact lenses and spectacles for the correction of presbyopia. The simplest lens design uses a meniscus shaped cavity within the lens to contain the liquid crystal. The design of such a lens is considered in detail, including the nematic alignment and electrodes materials. The organic transparent conductor PEDOT:PSS was used as both electrode and planar alignment. Four different configurations are considered, using both planar and homeotropic orientations with either homogenous or axial alignment. Controllable switching of the focal power was demonstrated for each mode and focal power changes of up to $\Delta P=3.3 \pm 0.2 D$ achieved. Such lens designs offer significant potential for a novel form of correction for this common visual problem.
\end{abstract}

Keywords: Liquid crystals, nematics, electro-optic effects, switchable contact lenses

1

Introduction:

Presbyopia is the visual disorder resulting from the reduction of natural accommodation which hampers functional near vision $[1,2]$. It is caused by the age-related hardening of the crystalline lens tissue in the eye and corresponding reduced elasticity of the accommodative mechanism that changes lens shape. Presbyopia affects almost all individuals from their mid-40s. Worldwide it was estimated that there were 1.272 billion sufferers of presbyopia in 2011 [3]. The population of presbyopia sufferers is growing strongly in developed counties where the average ages continues to increase. Age inevitably leads to presbyopia, but other risk factors include diabetes mellitus, vascular insufficiency, myasthenia gravis, anaemia, influenza and measles.

Given the obvious importance of good sight for daily life, this failure of the visual system is at best a marked inconvenience. However, even when presbyopia is corrected with currently available optical solutions it has a measureable impact in the quality of life of an order similar to treated hypertension $[4,5]$. Ten per cent of people report that they would trade $5 \%$ of their remaining life to be rid of the symptoms of presbyopia [5]. In a rare healthcare condition this would be noteworthy 
enough, but in a near-ubiquitous condition such as presbyopia, innovative and disruptive approaches to its correction promise widespread improvement to life quality. Treating presbyopia is therefore a worthy challenge that continues to become more relevant. It has no cure and the best treatment currently available is visual correction using spectacle lenses [2]. Correction for distance vision may also be necessary (or the wearers might prefer simultaneously clear distance and near vision), which is why bifocal or varifocal spectacles are used. A more discrete solution, such as a contact lens is desirable for those who do not wish to wear glasses, for cosmetic or other reasons. The simplest technique that is currently available is using lenses of differing focal powers in each eye [2]. Multifocal lenses are also available that focus light from both near and far objects onto the retina simultaneously [6]. Both techniques can be disorientating and it takes the user some time to train their brain to distinguish between the two images being focused simultaneously. However, not all wearers are able to adapt to either of these solutions. A contact lens that is automatically able to selectively switch between the different foci when required is beneficial as it would reduce this inconvenience and disorientation [6-8].

Liquid crystals (LCS) are already being investigated as a solution to making lenses $[9,10]$ and contact lens with electrically switchable focus [11]. This utilises the inherent birefringence of the liquid crystal to change the focal power of the lens through electrically controlled changes of the liquid crystal orientation, and hence refractive index. There are many different techniques that have previously been proposed for switchable LC lenses. This includes lens shaped LC layers, $[9,10]$ flat gradient index lenses $[12,13]$, diffractive lenses $[14,15]$ and flat lenses using inhomogeneous electric fields [16-19]. Recently, PMMA lens substrates have been used to fabricate switchable contact lenses [6]. The materials and manufacturing techniques used are comparable to those already used in conventional contact lenses $[6-8,20]$. A non-uniformly spaced cavity was created within the PMMA lensing substrates, with electrodes and alignment layers deposited onto their surfaces. The cavity between the lenses was filled with a nematic liquid crystal to form a single switchable contact lens, illustrated in figure 1 . The use of a curved substrates and variable cell spacing's are atypical for liquid crystal devices. However, forming a one- or two-chambered lens from the contact lens material, such as PMMA, keeps manufacturing difficulty to a minimum. Unlike LCD, contact lenses are fabricated singularly, which means that adaptation of the $L C$ fabrication processes for use with curved substrates is relatively straightforward, as described in this paper. So, it remains important to understand the maximum variation in liquid crystal thickness that is required to provide sufficient correction for the wearer.

A contact lens may need to provide correction for both distance and near vision. However, the condition of presbyopia represents a weakening of the focussing power of eye and can be corrected by $+0.75 \mathrm{D} \leq \Delta \mathrm{P} \leq+2.5 \mathrm{D}$ for the great majority of sufferers. If one of the indices of the liquid crystal (usually the ordinary refractive index $n_{0}$ for standard, calamitic liquid crystals) matches the index of the lens, then the substrates are arranged so that the net curvature of the system gives the correct lensing power for the distance vision, and the field on state for the liquid crystal gives $\Delta P$ $=+0.75 \mathrm{D}$ to +2.5 to help the natural eye attain the focus needed for short vision. The change in focal power $\Delta P$ from a thin lens is given by;

$$
\Delta P=\left(n_{1}-n_{2}\right)\left(\frac{1}{r_{1}}-\frac{1}{r_{2}}\right),
$$




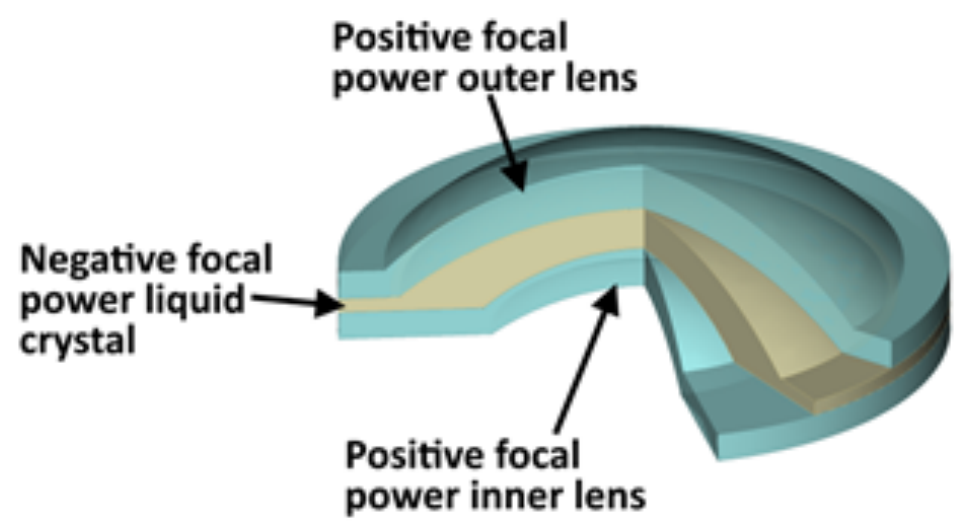

Figure 1: Schematic of the basic liquid crystal contact lens. In this example two positive focal power PMMA layers form a cavity with a negative focal power between them. Liquid crystal within this cavity allows the focal power of the device to be controlled by applying an electric field.

where $n_{1}=$ refractive index of medium around lens, $n_{2}=$ refractive index of the lens, $r_{1}=$ radius of curvature of the lenses lower surface and $r_{2}=$ radius of curvature of the lenses upper surface. A lens will have a positive focal power $(\Delta \mathrm{P}>0)$ if $r_{1}>r_{2}$ when $n_{2}>n_{1}$, or if $r_{1}<r_{2}$ when $n_{2}<n_{1}$. Likewise, the lens will have a negative focal power if $r_{1}<r_{2}$ when $n_{2}>n_{1}$. For a liquid crystal lens, the electrically induced change in refractive index is related to the birefringence $\Delta \mathrm{n}$. Usually, the ordinary index of the liquid crystal is matched close to that of the lens substrate (PMMA has a typical refractive index of 1.495). This means that the liquid crystal has limited additional optical effect in the states where the director is normal to the cavity surfaces (either field fully on, or off). Lensing is dominated by the difference in curvature between the outer and inner surfaces of the contact lens while in this mode. In this fashion, the contact lens can correct for long distance vision, and provide either highly positive or negative dioptre correction. Switching to the state where the light traversing the liquid crystal cavity in the lens experiences the extraordinary refractive index is only needed to correct near vision, and to augment the natural focussing ability that remains in the presbyopic eye. This is why correction of only $+0.75 \mathrm{D} \leq \Delta \mathrm{P} \leq+2.5 \mathrm{D}$ is needed for the liquid crystal element.

The geometry of the spherical LC cavity illustrated in figure 2 is calculated by the following equation [6]:

$$
\Delta Y=R_{1}-R_{2}-\sqrt{R_{1}^{2}-X^{2}}+\sqrt{R_{2}{ }^{2}-X^{2}}
$$

where $X$ is the radius of the active aperture (the human pupil is typically less than $2.5 \mathrm{~mm}[21]$ ), $R_{1}$ is similar to the anterior radius of the cornea (typically $7.8 \mathrm{~mm}$ [21]), $R_{2}=$ radius of the outer curvature of the liquid crystal cavity and $\Delta \mathrm{Y}$ is change in cavity spacing at aperture distance $\mathrm{X}$. The maximum spacing in a LC cavity is calculated by $h=h_{0}+\Delta Y$ (shown in figure 2), where $h_{0}$ is the minimum spacing to ensure manufacturability, optical performance and insulation of the electrodes on the upper and lower surfaces of the cavity (typically taken to be between $2 \mu \mathrm{m}<h_{0}<5 \mu \mathrm{m}$ ). Using a 
variable spacing would present problems with many electro-optic media because the applied field reduces as the space between the electrodes increases. However this is not an issue with nematic liquid crystals, since switching is governed by the Fréedericksz effect and is dependent on the applied voltage rather than field [22]. Ensuring that the maximum spacing of the LC cavity is kept as low as possible is important, because this ensures faster switching times, leading to a more comfortable user experience. A second reason for limiting the cavity spacing is due to the alignment degradation that occurs if the cell spacing is greater than $100 \mu \mathrm{m}$ (note, this limit is somewhat higher than for a typical uniformly spaced device, because of the influence of the surrounding well-aligned liquid crystal in the lower-spaced regions). This maximum spacing adds restrictions on the geometry of the LC layer, which in turn limits the maximum change in focal power, as given by equation 1. An LC with a high birefringence should be used to ensure that the required change in focal power occurs for the lowest maximum cavity spacing $h$. Furthermore, lower voltages would be needed to switch the lens to a moderate focal power when a higher birefringence $L C$ is used.

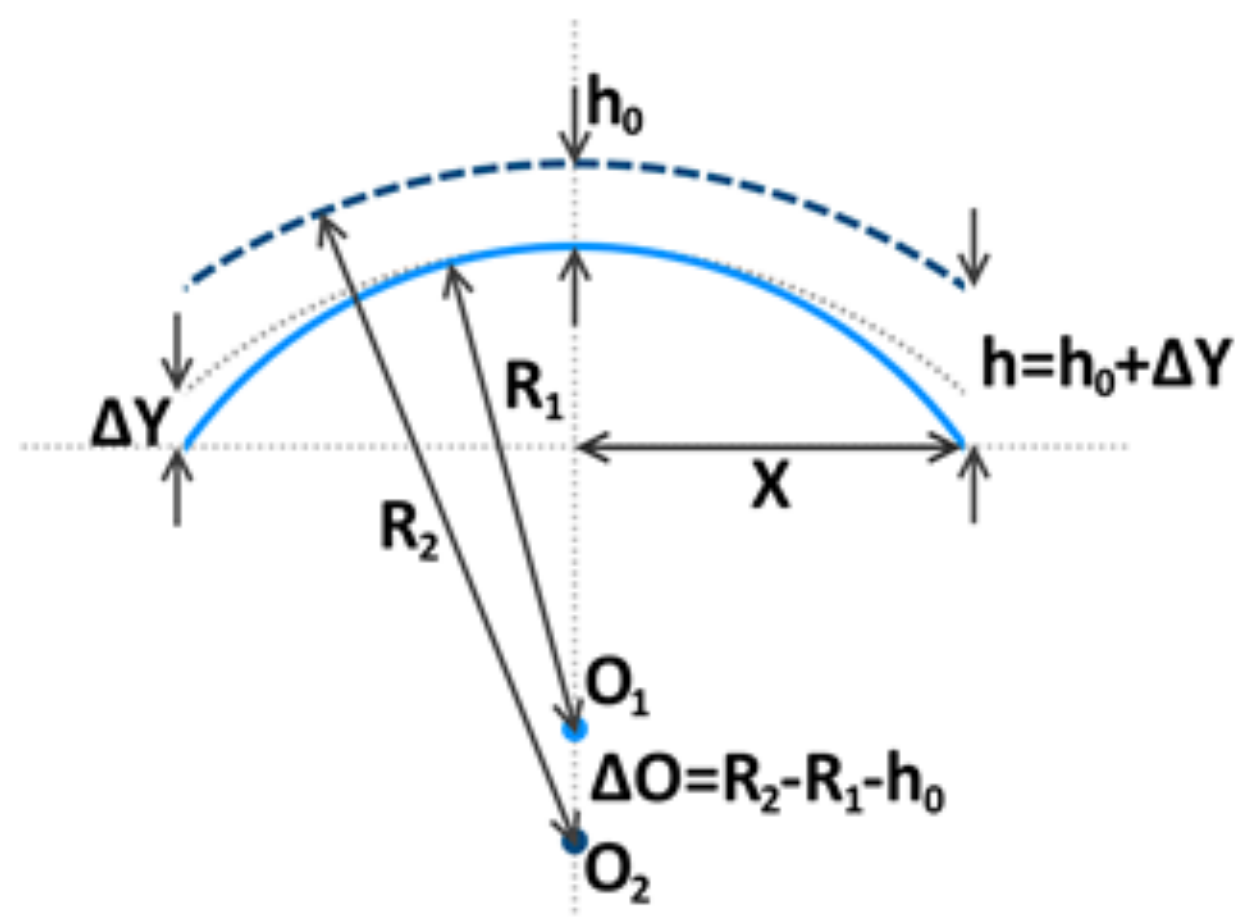

Figure 2: Diagram illustrating how the focal power is calculated using the lens equation.

Figure 3 shows the required change in cavity spacing across the width of the pupil ( $4 \mathrm{~mm}$ ) to cause $+1.5 \mathrm{D},+2.0 \mathrm{D}$ and $+2.5 \mathrm{D}$ of optical correction, as a function of the difference in refractive index for the ON and OFF states. If the voltage is sufficiently high, typically three or four times greater than the Fréedericksz threshold voltage (i.e. $3 \mathrm{~V}$ to $4 \mathrm{~V}$ ), the change in refractive index approaches the inherent birefringence of the liquid crystal, $\Delta \mathrm{n}$. Figure 3 shows the $\Delta y$ required for the change in focal power for target $\Delta P=1.5 \mathrm{D}, 2.0 \mathrm{D}$ and $2.5 \mathrm{D}$ as a function of $\Delta \mathrm{n}$. UV stable nematic liquid crystals with $0.24 \leq \Delta \mathrm{n} \leq 0.28$ are practicable, for which the target $\Delta \mathrm{Y}$ is only about $20 \mu \mathrm{m}$ to $25 \mu \mathrm{m}$. (The 
figure indicates the room temperature $\Delta n$ values for the liquid crystals E7 and MLC-2081 for context). For a typical eye with reasonable values for $\Delta \mathrm{Y}$ and $\Delta \mathrm{n}$, the data is approximately given by:

$$
\Delta Y=3.32 \frac{\Delta P}{\Delta n}
$$

For high spacing's, only the OFF time $\tau_{\text {OFF }}$ is relevant. Ignoring flow, this is given by [19]:

$$
\tau_{O F F}=\frac{\gamma_{1} h^{2}}{\pi^{2} K}
$$

where $\gamma_{1}$ is the rotational viscosity of the LC director and $\mathrm{K}$ is the geometry dependent elastic constant, taken to be the average of splay $K_{11}$ and bend $K_{33}$ elastic constants for the devices studied in this work. Equation 4 predicts that the response time for optimised lenses should be about $0.5 \mathrm{~s}$, which is discussed further in the results section. Furthermore, it is envisaged that faster response times are readily achievable using higher elastic constant mixtures (given that high $\Delta \mathrm{n}$ LC materials tend to have correspondingly higher $\gamma_{1}$ ), with only a minor cost in operating voltage.

In summary, nematic liquid crystals readily offer the means for switching $+2.0 \mathrm{D}$ at only a few volts, using a simple lens shaped cavity within a standard contact lens. In this paper, we will discuss the different alignment geometries, electrode materials and fabrication techniques used, to help optimise the switchable contact lens performance.

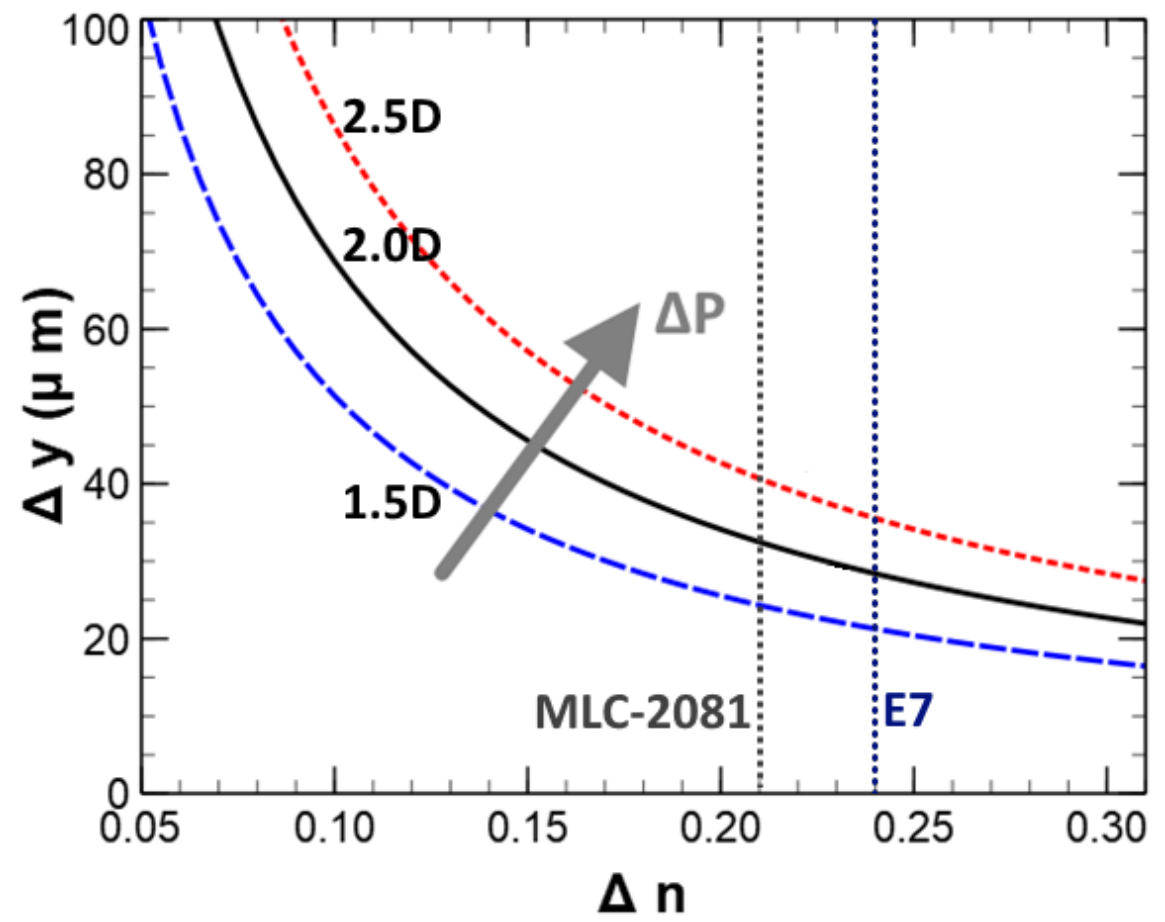

Figure 3: The maximum difference in cavity spacing change $\Delta Y$ that is required when targeting focusing powers of $\triangle P=1.5 D, 2.0 D$ and $3 D$ as a function of the change in refractive index between switched and un-switched states. These curves are calculated using equation (3). The birefringence of E7 and MLS-2081 are shown in this plot for comparison. 
There are four basic lens design modes, depending on whether the liquid crystal cavity forms a positive or negative meniscus lens, and whether the quiescent, field-off alignment configuration of the liquid crystal is vertically aligned (VAN) or planar aligned (PAN), as shown in figure 4. In each case, the lens focal power is changed by electrically reorienting the LC director to be either parallel or perpendicular to the surface, to give either a positive or negative optical change, $\Delta \mathrm{P}$, defined with respect to the change from the field off to field on states as shown. This is controlled by surface alignment and switching of the liquid crystal by applying an electric field across the cavity from electrodes deposited onto the opposing internal surfaces. The design choice depends upon whether a positive or negative $\Delta \mathrm{P}$ is required; that is, whether or not the near vision correction is provided in for the field OFF or field ON states. Presbyopia always requires a positive correction $\triangle \mathrm{P}$ for short distance vision. The lens may be considered more power efficient if designed to provide distance vision without operating voltage, as this operation mode is more likely be the lens's more frequent setting. Using an LC with a positive dielectric anisotropy would then require its cavity within the lens to have a negative focal power. Likewise, using a LC with a negative dielectric anisotropy requires the LC layer to have a positive focal power. This occurs for the positive meniscus lens in a VAN mode with a positive $\Delta \varepsilon$ liquid crystal, or for the negative meniscus lens in a PAN mode with a negative $\Delta \varepsilon$ liquid crystal; options A and D in figure 4, respectively.

The positive meniscus lenses have the minimum cavity spacing at the edges of the pupil. This means that switching from the OFF to ON state occurs from the edge of the pupil inwards, contrary to the negative meniscus options. Given that the response time is proportional to the cavity spacing, $h$, squared through equation (4), which means that switching of the lens is initially fast but slows markedly at the centre. Therefore, the optical correction is effectively much faster when the positive meniscus lens is chosen, because the strongest optical change corresponds to the part of the lens with lowest spacing. That is, the majority of the $\pm 2 \mathrm{D}$ correction when switching off would happen much faster than the full response time. We predict that use of mode A would be more comfortable for the user for this reason and a target full change in focus in under $0.5 \mathrm{~s}$ for an optimised lens is more than adequate (discussed in greater detail in the results section).

The variable spacing leads to a switching soliton that moves from the fast to slow regions as the director responds to the applied voltage. This soliton may influence the director alignment of the final switched state. In practice, conflict with the local surface variations can lead to domains forming separating areas of opposing tilt. Such domain cause scattering, which are distracting to the user and detrimental to the lens performance. For example, consider the negative meniscus homeotropic alignment of design $\mathrm{C}$. The soliton begins at the point of the lowest spacing at the lens symmetry axis. For homeotropic boundaries, switching to the low tilt state is degenerate, and usually subject to random surface variations. For homeotropic lenses of design C, switching starts at a single point in the cell centre, rather than the circumference of starting points that occurs with lenses of design A. This is likely to lead to less degeneracy of switching since the induced director tilt is also influenced by the flow effect of the switching soliton. Such domains can also be formed in the planar aligned cases of $B$ and $D$. This can be reduced using a polyimide with a pretilt $>\tan \left(\Delta Y / X_{\max }\right)+$ $2^{\circ} \approx 3^{\circ}$ to ensure that the alignment effect dominates, or by designing lenses with an axial alignment symmetry (i.e. either axial or radial alignment). This results that there are four different alignment modes with uniform director profiles that can be chosen. An important part of the work reported 


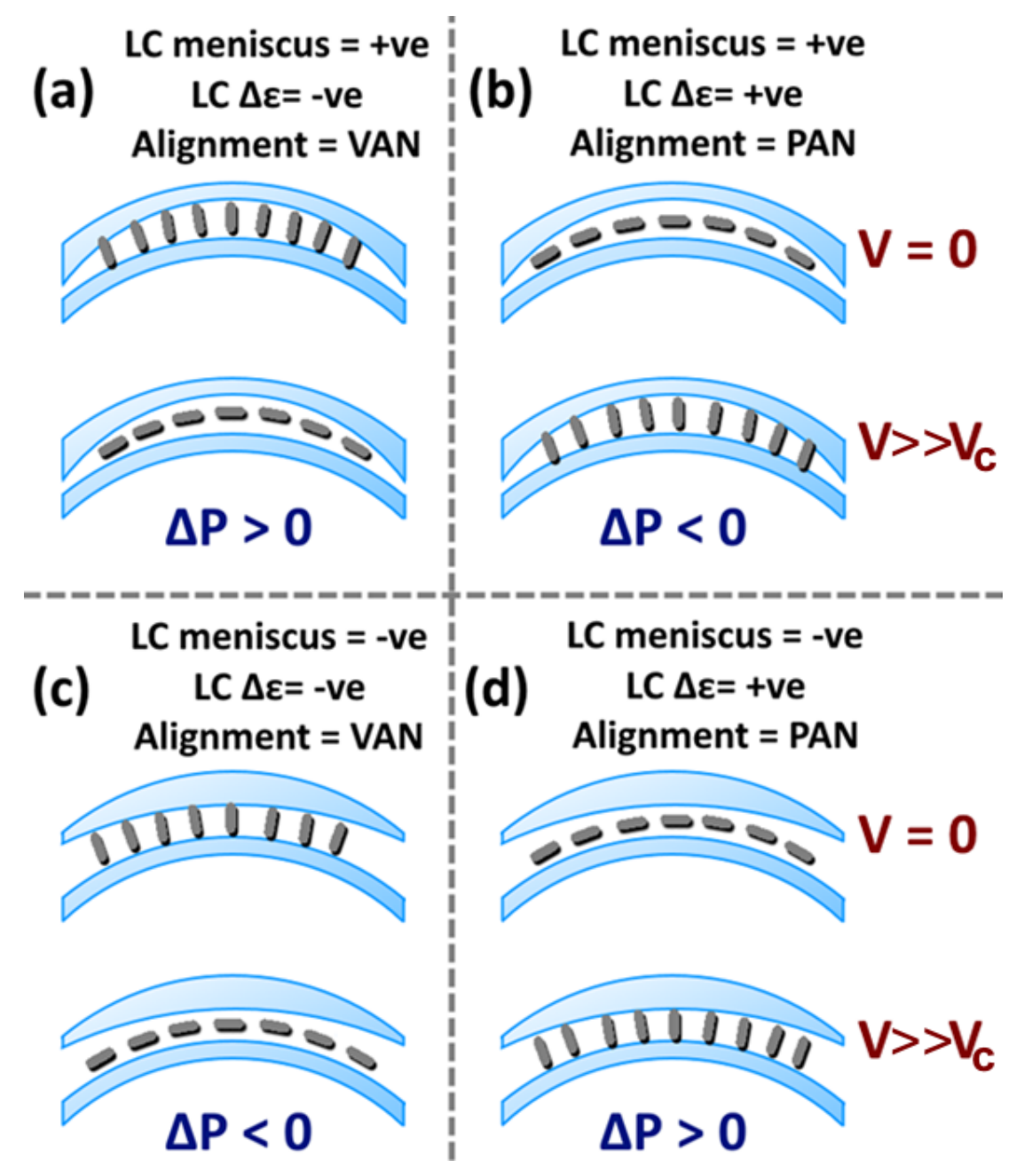

Figure 4: Positive and Negative meniscus lenses operating in either homeotropic or planar aligned modes. The correct $L C$ cavity shape must be pared with the appropriate $L C \Delta \varepsilon$ for the desired optical mode when the lens is ON or OFF.

here is to distinguish between these alignment modes and to determine whether they have any impact on lens electro-optical performance. These alignment modes are; planar homogeneous (PH), planar axial (PA), homeotropic homogeneous $(\mathrm{HH})$ and homeotropic axial (HA). Note, the homeotropic geometries differs from conventional homeotropic alignment only in the use of some rubbing (or other such symmetry breaking, including photo-alignment) to ensure that switching of the director is not degenerate but aligned with respect to the axis of symmetry. In practice, this might result from a negligible pretilt away from the surface normal. Figure 5 illustrates how LC's with a positive $\Delta \varepsilon$ (using alignment geometries $\mathrm{PH}$ and $\mathrm{PA}$ ), will orientate from being parallel to the lens surface to perpendicular when the potential is greater than the Fréedericksz transition threshold voltage $V_{c}$. Likewise, lenses that $\mathrm{LC}^{\prime} s$ with a negative $\Delta \varepsilon$ (using alignment geometries $\mathrm{HH}$ and $\mathrm{HA}$ ), will orientate from being perpendicular to parallel along the surface when switching above $V_{c}$. The orientation of the LC when director is parallel to the surface is pre-determined by the rubbing direction during sample preparation (discussed in detail later). 
The most common electrode used in LC lens experiments to date has been indium tin oxide (ITO) [6-8] sputtered onto the PMMA. This material was selected previously because of its relatively high conductivity and optical transparency, and compatibility with liquid crystal alignment layers. Although it is often quoted that Indium is becoming more expensive due to its increasing demand in devices [20], a more important issue is that the conductivity and absorption spectra of ITO is dependent upon its film thickness [21,22]. This is difficult to control when sputtering onto a curved substrate, such as the contact lenses used here. Previously, the PMMA lenses required a spin-onglass coating to improve the adhesion of ITO to its surface, which increases manufacturing steps and costs. ITO is also a problematic material when deposited onto flexible substrates due to its brittleness $[23,24]$. This was not a substantial problem for PMMA hard contact lenses which are relatively rigid. However, it will become a more important issue when a flexible and more comfortable soft-lens substrate, such as a hydrogel, is used once the lenses become commercialised. Furthermore, an additive manufacturing approach has enormous advantages over sputtering for simple low-cost fabrication on the laboratory scale. There have already been several studies into replacing ITO for use in devices with flexible substrates. This includes using conductive polymers $[25,26]$, metallic nanowires [20,27], carbon nanotubes [28,29] and graphene [16,30]. We chose Poly(3,4-ethylenedioxythiophene) polystyrene sulfonate (PEDOT:PSS) as it is a flexible conducting polymer that can be deposited using simple solution processing techniques (such as spin-coating, screen printing and inkjet printing). The surface roughness of PEDOT:PSS is typically $<10 \mathrm{~nm}$ when deposited using either spin-coating [34] or inkjet printing [35] techniques. This is an order of magnitude less than the aberrations on the surface of the contact lens which have a manufacturing tolerance of $<500 \mathrm{~nm}$ (which is typical of commercial contact lenses) [6]. Therefore, the addition of PEDOT:PSS does not change the optical quality of the lenses when compared to the manufacturing tolerances of PMMA. PEDOT:PSS has already been used in a wide range of opto-electronic devices such as OLED's [36], solar cells [32] and liquid crystal cells [28,29]. It has also been used previously in larger lenses that are able to switch focal power [38-40] and also used in single pixel contact lenses. Those single pixel contact lenses were designed to change optical contrast, instead of their focal power [41-43]. To our knowledge, this is the first time PEDOT:PSS has been used as an electrode to switch the focal power of a LC contact lens. Another benefit of using PEDOT:PSS is that it's polymer layer can be rubbed and used for LC planar alignment. This reduces the number of manufacturing steps and eliminates the need for compatible materials and processing techniques for a multilayered system. 

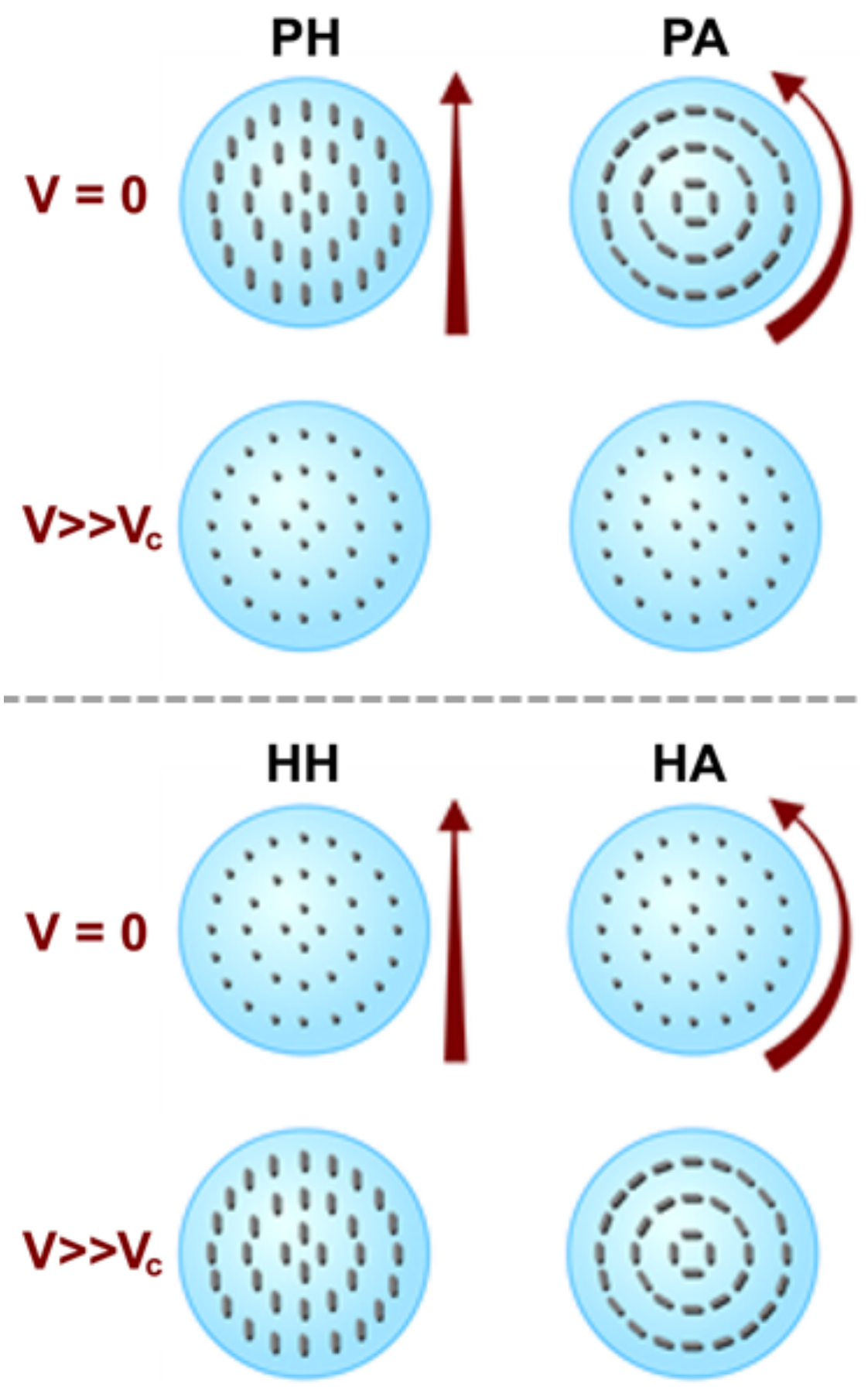

Figure 5: Illustration of the alignment modes tested to determine whether they change the optical properties of the lenses. The modes are planar homogeneous (PH), planar axial (PA), homeotropic homogeneous $(\mathrm{HH})$ and homeotropic axial (HA). The red arrows indicate the direction of the alignment applied to the lenses. Changes in the $L C$ 's director are shown when no Voltage is applied $(V=0)$ and above the critical threshold voltage $V_{c}\left(V>>V_{c}\right)$ over the cell. 


\subsection{Sample construction:}

The samples in the current work were constructed by machining two halves of the contact lens from ophthalmic grade PMMA blocks using an Optoform 30 contact lens lathe (Sterling, Florida, USA). For the test lenses, minimal optical correction for short sightedness was included, so that the first and second substrates were both uniform, and with radii of $7.8 \mathrm{~mm}$ for the inner eye, and the curvature of the cavity for the outer element. Both LC cavities in the two planar lenses (PH and PA) had a lower and upper radius for $7.8 \mathrm{~mm}$ and $8.5 \mathrm{~mm}$ respectively. The radius of curvature to form the LC cavities for both the homeotropic lenses ( $\mathrm{HH}$ and $\mathrm{HA}$ ) were $7.9 \mathrm{~mm}$ and $8.5 \mathrm{~mm}$ respectively due to slightly different lens designs.

The construction of the LC contact lens is illustrated in figure 6(a). Both sides of the PMMA lens were cleaned by sonication for 30 minutes in 1:4 Decon 90:deionised water and then rinsed by sonication for 30 minutes in deionised water. The lenses were dried using compressed air and outer surface was placed onto a spin-coater. IPA was deposited onto the lenses while it was spinning at $4.0 \mathrm{krpm}$ for approximately 30 seconds. Two different techniques where tested for coating the PMMA lenses with PEDOT:PSS (Sigma-Aldrich). Both the of the lenses that used planar alignment (PH and PA) used an 'adhesion layer' of $40 \%$ w.t. Silquest A-287 silane (Momentive) mixed with PEDOT:PSS and spin-coated onto the internal facing sides of the lens at $3.0 \mathrm{krpm}$ for 30 seconds. This adhesion layer was baked at $55 \pm 5^{\circ} \mathrm{C}$ for 10 minutes and then cooled to room temperature. A 'conduction layer' of 7\% w.t. Dimethyl sulfoxide (DMSO) (Merck) in PEDOT:PSS was spin-coated on top of the 'adhesion layer' at $1.5 \mathrm{krpm}$ for 1 minute. Again, the conduction layer was dried by baking at $55 \pm 5^{\circ} \mathrm{C}$ for 15 minutes and then cooled to room temperature. The homeotropic lenses $(\mathrm{HH}$ and HA) were prepared by passivating the PMMA surface with 10 minutes of UV ozone exposure using a UVOCS T10X10/OES. The PEDOT:PSS layer was spun directly onto the passivated surface at $2 \mathrm{krpm}$ for 30 seconds. This initial layer was baked for 10 mins at $55 \pm 5^{\circ} \mathrm{C}$ for $10 \mathrm{mins}$ and then allowed to cool back to room temperature. A second PEDOT:PSS layer was added by spin-coating onto the previous layer at $2 \mathrm{krpm}$ for 30 seconds. The second layer was baked for $15 \mathrm{mins}$ at $55 \pm 5^{\circ} \mathrm{C}$. All of these processes were repeated for the opposing lens substrate so a complete contact lens could be constructed.

The orientation of the director was applied to the both the planar aligned lenses (PH and PA) by rubbing directly into the PEDOT:PSS layer. Both of the PEDOT:PSS layers need to be rubbed to form anti-parallel alignment for the PH and PA alignment modes. Care must be taken when aligning the $\mathrm{PH}$ layers as their linear direction can easily be misaligned during assembly. This is mitigated when using PA or HA alignment, as the circular symmetry then means that the sample cannot be misaligned when rotating the sample. The PA alignment was prepared by placing the lens onto a spin-coater and applying rubbing cloth to the PEDOT:PSS layer as the sample rotates. Homeotropic alignment for the $\mathrm{HH}$ and HA alignment layers was applied by spin-coating SE-1211 polyimide (Nissan Chemical) onto the PEDOT:PSS electrode layer. This polyimide layer was then hardened by baking for 3 hours at $50^{\circ} \mathrm{C}$. The $\mathrm{HH}$ alignment was applied by rubbing the polyimide layer linearly. $\mathrm{HA}$ alignment was applied using the same spin-coater mounted technique as PA. Both the HH and HA alignment modes only required one side of the lens cavity to be rubbed. This was shown to be 
sufficient to predetermine the alignment direction when switching. Also, it is not possible to misalign the homeotropic samples when using this technique, as only one side requires rubbing for both $\mathrm{HH}$ and HA. Rubbing was preferentially applied to the convex surface for both the HH and HA lenses prepared, as it was easier to prepare than using the concave side.

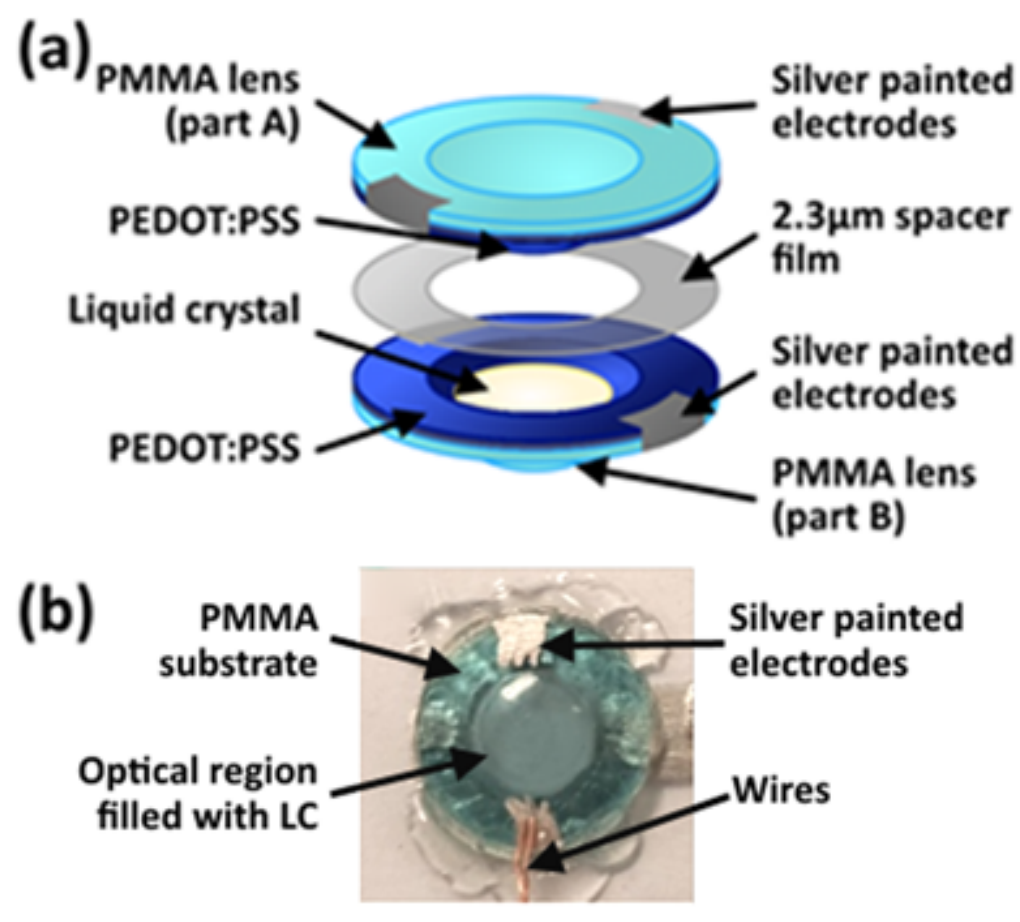

Figure 6: (a) Illustration of the parts used to construct the liquid crystal contact lenses. The inner surfaces of the PMMA lens substrates are coated with the electrode/alignment layer PEDOT:PSS. Short circuiting was prevented by using a $2.3 \mu \mathrm{m}$ spacer film. Electrical contact between external wires and cell was improved by using silver paint. Each cell was filled with the required LC before sealing it with UV glue. (b) Completed liquid crystal lens with wires attached leading to a signal generator.

Conductivity between the PEDOT:PSS electrode layers and the connecting wires was improved by applying silver paint to the edges of the lens. A $2.3 \mu \mathrm{m}$ mylar spacer film (therefore, $h_{0}$ $=2.3 \mu \mathrm{m}$ from figure 2) was inserted between the two halves of the contact lens to prevent the device from short circuiting. The planar and axial lenses were assembled by filling the concave surface of the upper lens with the appropriate LC.

The LC chosen for our lens with planar and axial alignment was E7 due to its large birefringence $(\Delta n=0.24)$ and high degree of characterisation in the literature. A larger birefringence increases the switchable focal power range, as discussed previously. The largest change in focal power was predicted as 2.53D, using equation 1 . The negative dielectric LC chosen for the homeotropic cell was MLC-2081 $(\Delta n=0.210)$, which was used by Syed et. al. in their similar contact lens with ITO electrodes [8]. The largest change in focal power for these lenses was predicted to be 1.88D. The two halves of the lenses were assembled after filling the cavity with LC. UV glue was 
applied to the outer rim of the lenses and cured to secure the final device. This was then mounted onto a glass substrate and wires were attached so the lens could be tested (shown in figure 6(b)).

\subsection{Lens characterisation:}

A DM2500P polarizing microscope (Leica Microsystems, UK) was used to check the quality and alignment of the LC contact lenses. Figure 7 shows cross polarization microscopy images of both planar homogeneous (PH) and axially (PA) rubbed contact lenses without applied field. The planar lens microscopy images illustrate how the PEDOT:PSS layer is capable of functioning as an alignment layer when rubbed. Planar alignment appears dark when orientated parallel to either of the polarisers and brightest when the rubbing direction is orientated $45^{\circ}$ between the polarisers. A Maltese-cross pattern appears when viewing the axial lens between cross polarisers, due to the axial alignment symmetry of the LC director field. Rotating the axial sample does not change the cross pattern as the geometry is circular.
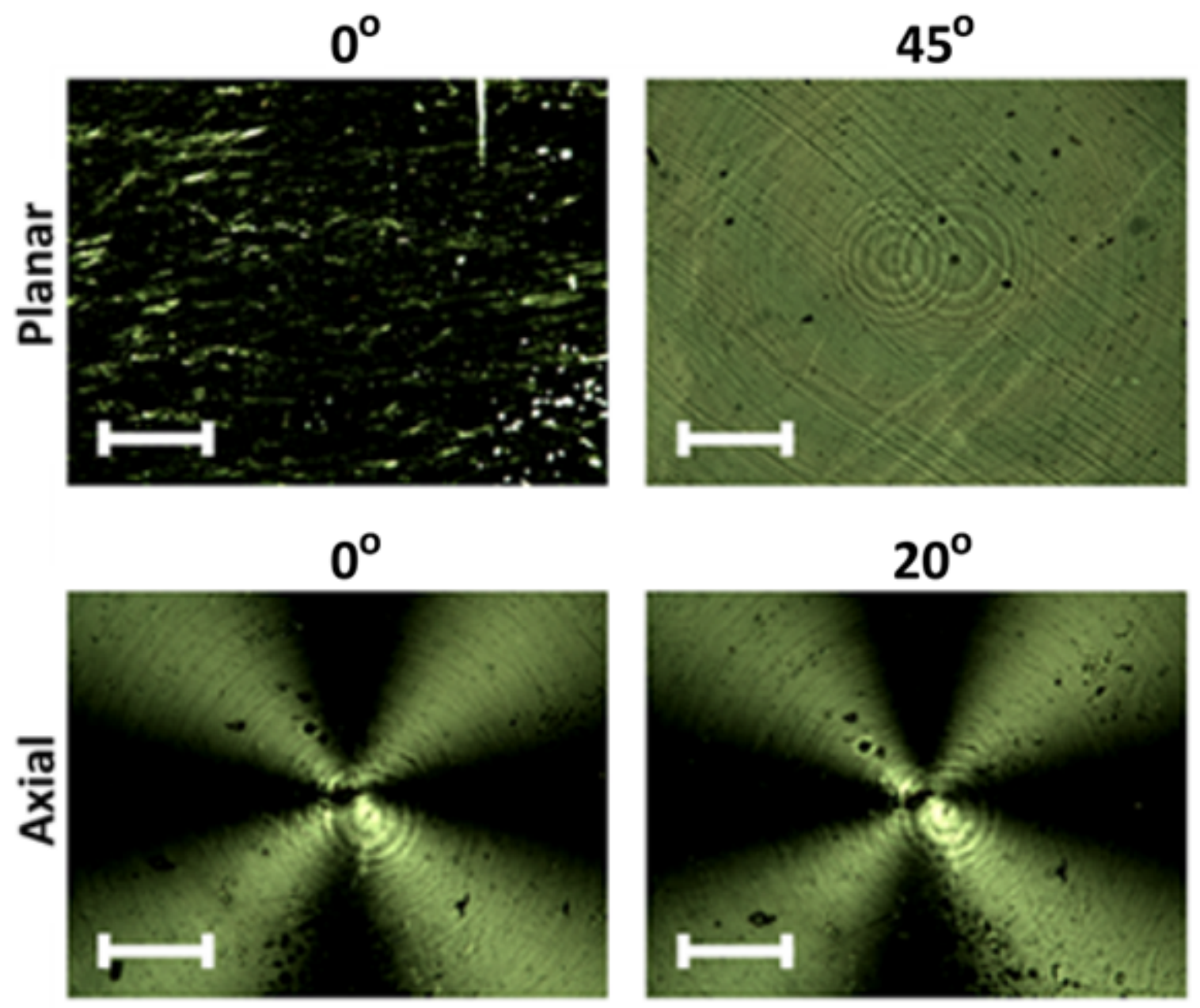

Figure 7: Polarizing microscopy images of planar, axial and homeotropic lenses. Both sets of images demonstrate that PEDOT:PSS is able to function as an alignment layer. The image is dark/bright when the director is parallel $/ 45^{\circ}$ with respect to the either of the microscope polarisers. A Maltesecross image occurs for axial alignment due to the director rotating around the centre of the lens. The scale bar for all images is $250 \mu m$. 
The focal power and optical quality of the contact lenses were measured using a purpose built optical set-up, using an expanded $10 \mathrm{~mW} 589 \mathrm{~nm}$ laser as the light source (illustrated in figure 8(a)). Planar aligned lens experiments had previously used a linear polariser to preferentially select the focal point from the $n_{e}$ refractive index component of the $L C[6-8,20]$. Preferential selection of the $n_{e}$ focal point is more challenging with axial alignment due to the director rotating around the centre of the lens. A slit was used to remove the majority of the LC in the lens that was not aligned parallel with the linear polariser. The remaining, predominantly parallel, LC in the axial lens was illuminated by the linearly polarised beam path (illustrated in figure $8(\mathrm{~b})$ ). A plano-convex lens was used to focus the beam onto a BC106-VIS CCD beam profiler (Thorlabs, New Jersey, USA) which had a pixel size of $6.47 \mu \mathrm{m}$ and a capture area of $1360 \times 1024(8.8 \mathrm{~mm} \times 6.625 \mathrm{~mm})$. The planar lenses used a $4 \mathrm{D}$ plano-convex lens, whereas the homeotropic lens required a $10 \mathrm{D}$ plano-convex lenes. This was to accommodate for the small changes in the PMMA substrates and the differing LCs used in the planar and homeotropic devices.

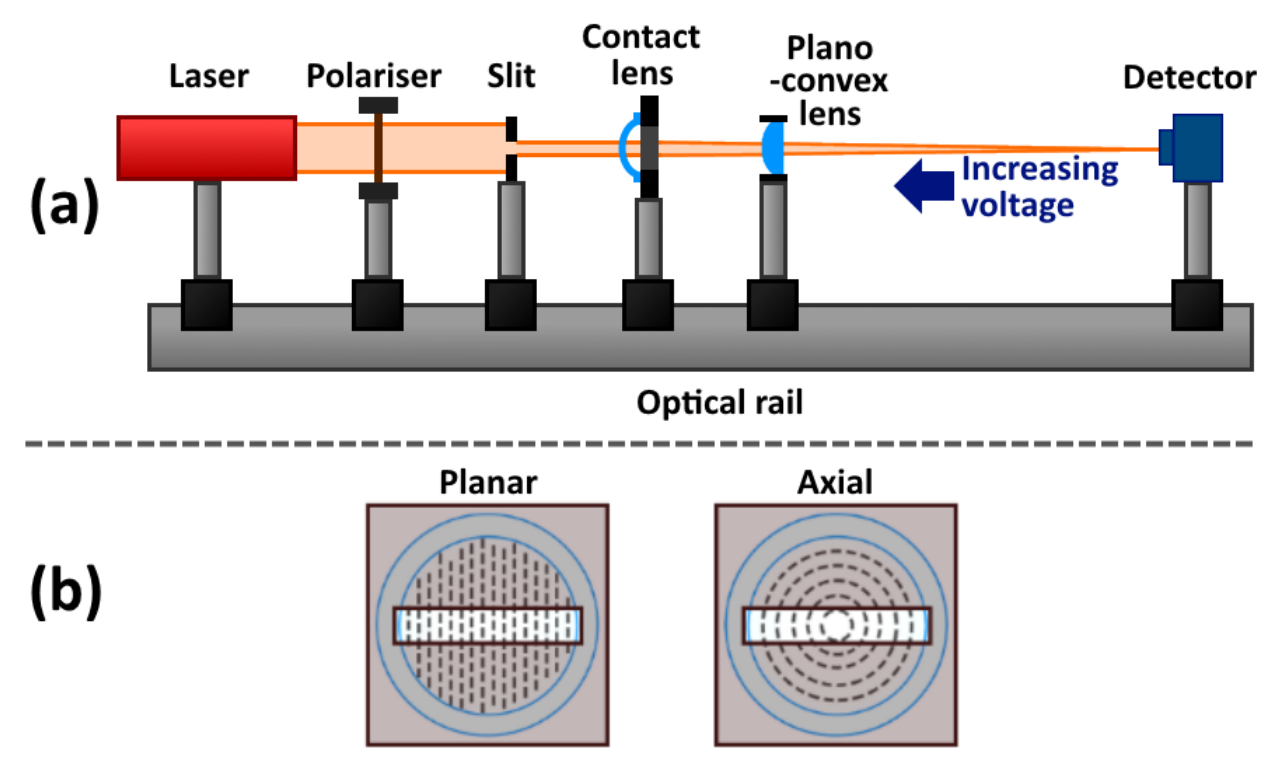

Figure 8: Illustration of the optical set-up used to measure the focal power and quality of the liquid crystal contact lenses is shown in panel (a). The detector needed to be moved towards/away from the lens to measure the focal point when increasing the applied potential over a liquid crystal with positive/negative anisotropy respectively. A slit (width $=460 \pm 30 \mu \mathrm{m}$ ) was used to measure a section of the axial aligned lens that is comparable to a planar lens geometry, which is illustrated by the diagram shown in panel (b).

A sinusoidal signal with a frequency of $23 \mathrm{kHz}$ was applied to the electrodes on the lens using an Agilent 33500B series signal generator. The potential was incrementally increased while the optical response on the beam profiler was analysed. All lenses showed a change in focal power after switching above the Fréedericksz transition. The change in focal power was calculated by repositioning the detector to the focus where the beam profile's maximum intensity occurred. This 
measurement was used with the following equation to calculate the focal power of the contact lens [44]:

$$
P=\left[d_{[C-P]}-\frac{f_{P} d_{[P-D]}}{d_{[P-D]}-f_{P}}\right]^{-1}
$$

where $d_{[C-P]}=$ distance between contact lens and plano-convex lens, $d_{[P-D]}=$ distance between plano-convex lens and detector and $f_{P}=$ focal point of plano-convex lens. A time delay was included when the applied voltage was increased and when the measurements were made. This was to ensure that the liquid crystal layer had re-orientated to an equilibrium state during the measurements. The waiting duration for the planar and axial lens measurements was 2 minutes, whereas a longer period of 5 minutes was chosen for the slower homeotropic lenses.

The ON and OFF switching times for the lenses were measured using a photodiode mounted onto the polarising microscope. Changes in optical intensity were measured through an $x 5$ magnification objective lens, which had a field of view diameter of $2.2 \pm 0.1 \mathrm{~mm}$. Switching times with respect to different voltages were measured in order to assess the performance of the lens at different power modes.

\section{$4 \quad$ Results}

All lenses showed changes in refractive index when viewed under cross polarisers and when sufficient potential was applied, shown in figure 9. The planar lens showed large uniform coloured areas when viewed at $45^{\circ}$ with respect to polariser and analyser angle and switched past the Fréedericksz transition figure 9(a)). This is due to the refractive index of the cavity changing as the as the LC re-orientates from being parallel to perpendicular with respect to the surface. The axial lens also shows changes in refractive index, with the Maltese cross pattern being retained to high switching voltages (figure 9(b)). Reverse tilt domains were observed in both the simple and axial planar lenses, but caused more deleterious scattering in the simple planar devices. However, these domains no not occur when using homeotropic alignment (figures 9(c) and 9(d)), but their homogeneous and axial alignment is apparent when they are being used.

The focal power of the PH and PA contact lenses had both increased when the applied potential was raised, shown in figure 10(a). Alternatively, the magnitude of the focal power had decreased for the $\mathrm{HH}$ and $\mathrm{HA}$ lenses when increasing the applied potential (fig 10(b)). This was due to all lenses having a negative meniscus LC cavity, which corresponds to the situations discussed previously figure $4(\mathrm{c})$ and $4(\mathrm{~d})$. Therefore, the pre-set for reading mode is $\mathrm{ON}$ when using PH or PA alignment and OFF when using $\mathrm{HH}$ and $\mathrm{HA}$ alignment. In practice, changing the lens shape of the LC layer from concave to convex (designs A and B) may be preferable for the particular task, but is yet to be studied.

The $\mathrm{PH}$ and $\mathrm{PA}$ lenses had the largest measured changes in focal power, tending to saturate above about $4 \mathrm{~V}$. At $6 \mathrm{~V} R \mathrm{RMS}$, the focal powers were $\Delta \mathrm{P} \approx 2.48 \pm 0.13 \mathrm{D}$ and $\Delta \mathrm{P} \approx 3.27 \pm 0.20 \mathrm{D}$ for simple planar and axial planar, respectively. It was previously calculated that the maximum change in focal power possible with E7 using the specified lenses was 2.53D. However, even a small $2.5 \%$ change in the radius of curvature in one of the lenses that forms the LC cavity is enough to increase 


\section{(a)}

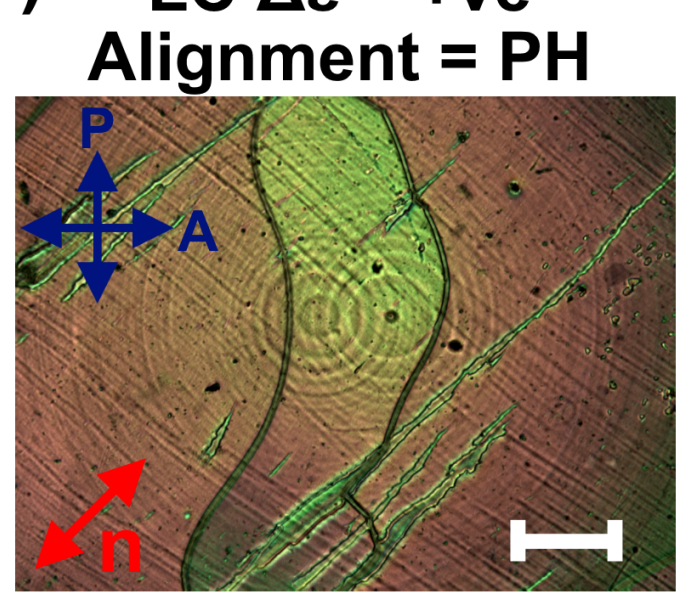

(c)

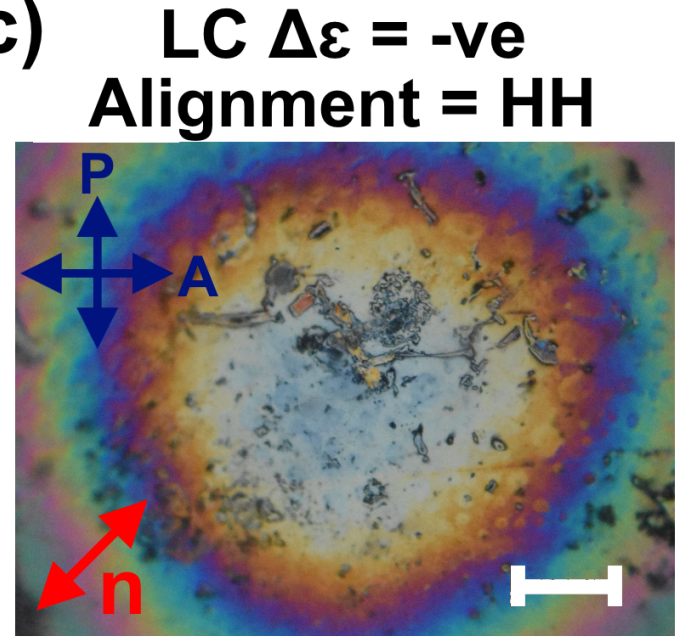

(b) LC $\Delta \varepsilon=+\mathrm{ve}$ Alignment $=$ PA

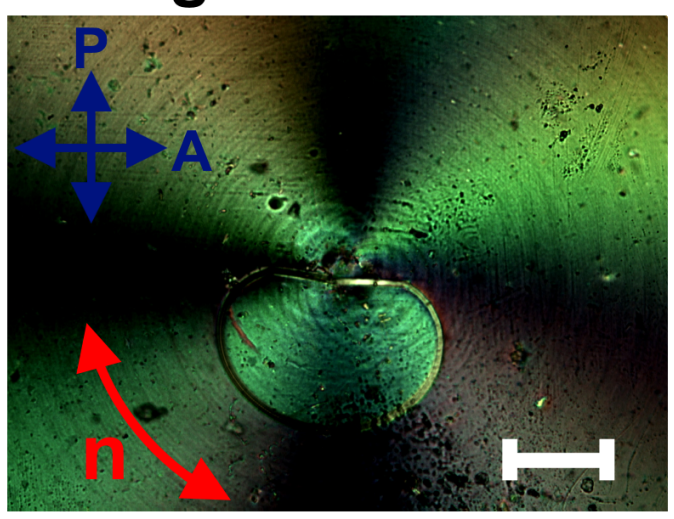

(d) LC $\Delta \varepsilon=-$ ve Alignment $=\mathrm{HA}$

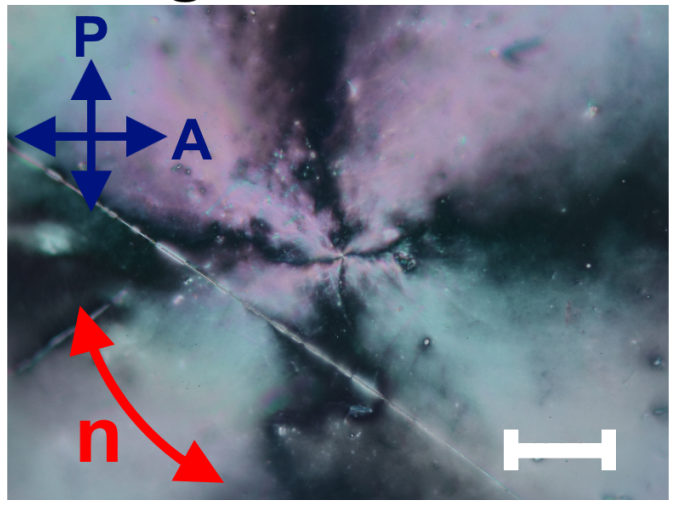

Figure 9: Cross polariser microscopy images of lenses that as 'switched' by applying 4.24V $V_{R M S}$ to the contact lenses. Domains were observed in only the LC lenses filled with $E 7(\Delta \varepsilon>0)$, shown in captions a) and b). Maltese cross patterns occur from the circular geometry of axial alignment, shown in captions b) and d). The scale bars micrographs are all 250 $\mu \mathrm{m}$. Directions of the polariser, analyser and director are noted by $P, A$ and $n$ respectively.

the change in focal power to $>3.3 \mathrm{D}$. This is illustrated in figure 11, where change in focal power is calculated with respect to changes in the LC cavities outer radius $r_{2}$ (the inner radius $r_{1}$ was kept constant at $7.8 \mathrm{~mm}$ ) using equation 1 . A larger outer radius increases the change in focal power, as well as the largest spacing in the lens $\Delta Y$. These small increases in radii can be caused during lens assembly, as the substrates are flexible enough to bend when too much pressure is applied.

The $\mathrm{HH}$ and $\mathrm{HA}$ lenses both began to saturate at $>6 \mathrm{~V}$ with their maximum changes in focal powers being $\Delta \mathrm{P}_{\mathrm{HH}}=1.52 \pm 0.25 \mathrm{D}$ and $\Delta \mathrm{P}_{\mathrm{HA}}=1.92 \pm 0.26 \mathrm{D}$ respectively. These measurements were above and below expected change in focal power were calculated to be $1.88 \mathrm{D}$. It is likely that the 
variations are due to discrete changes in the lens shape during the fabrication process, as discussed earlier.

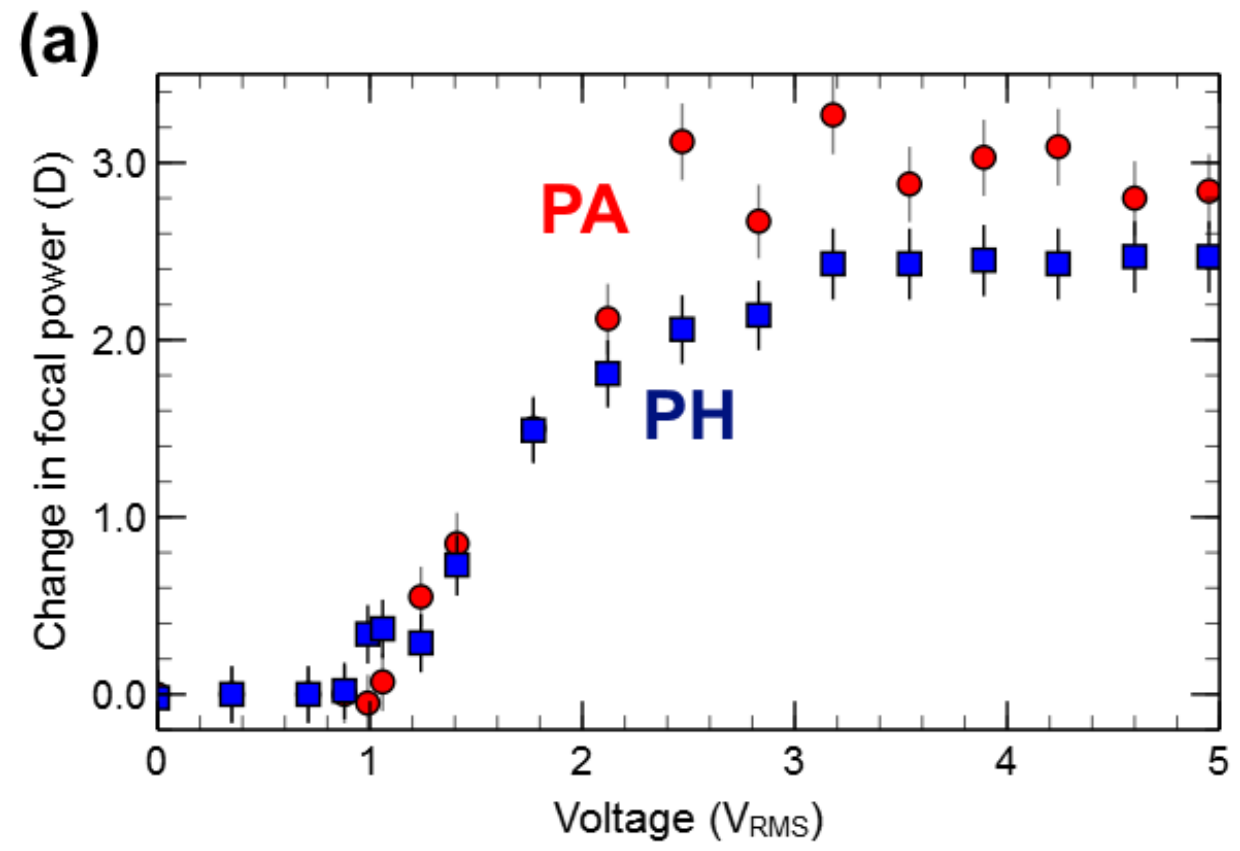

(b)

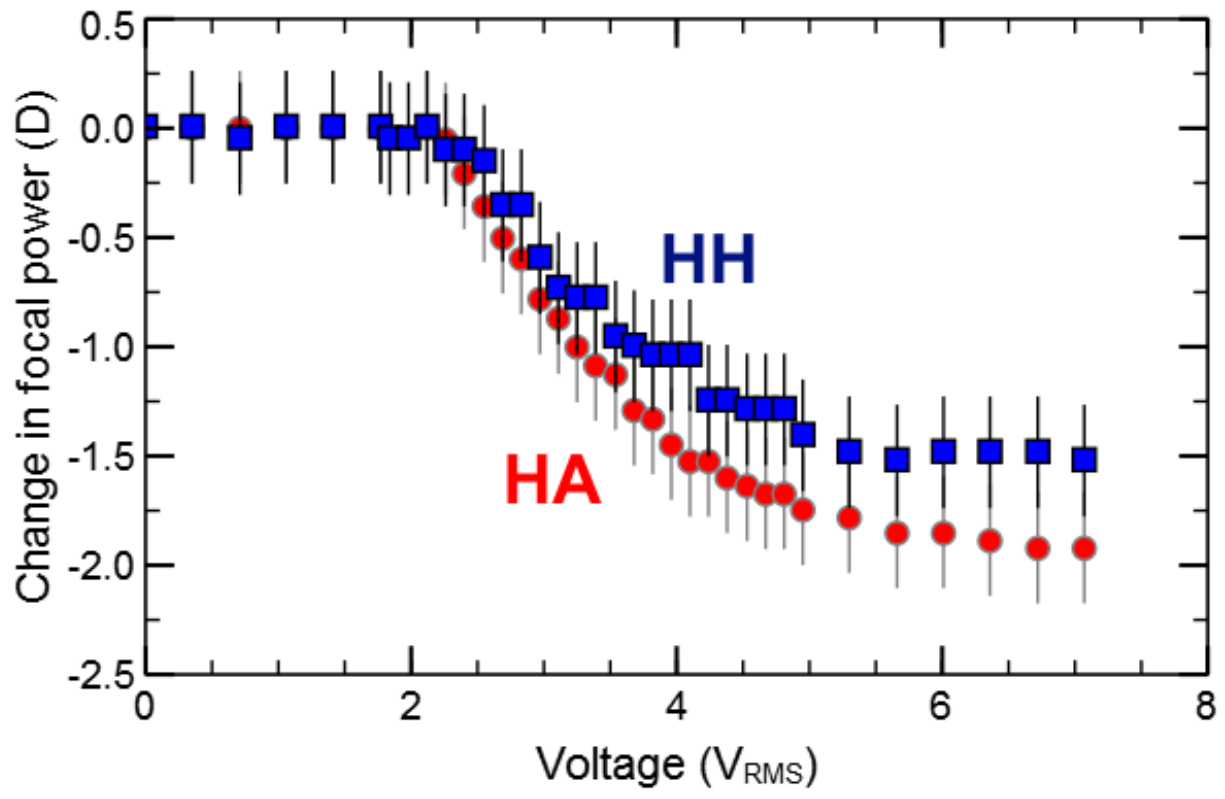

Figure 10: Panel (a) shows that the focal power increased for both PH (blue squares) and PA (red circles) alignment modes were increasing with respect to voltage once past the Fréedericksz transition. Panel (b) shows the focal power of the homeotropic lens degreasing as the voltage is increased for both HH (blue squares) and HA (red circles) alignment modes when greater than the Fréedericksz transition. The error bars for all data points were calculated by the propagation of uncertainty for the position of the optical components during measurements. 


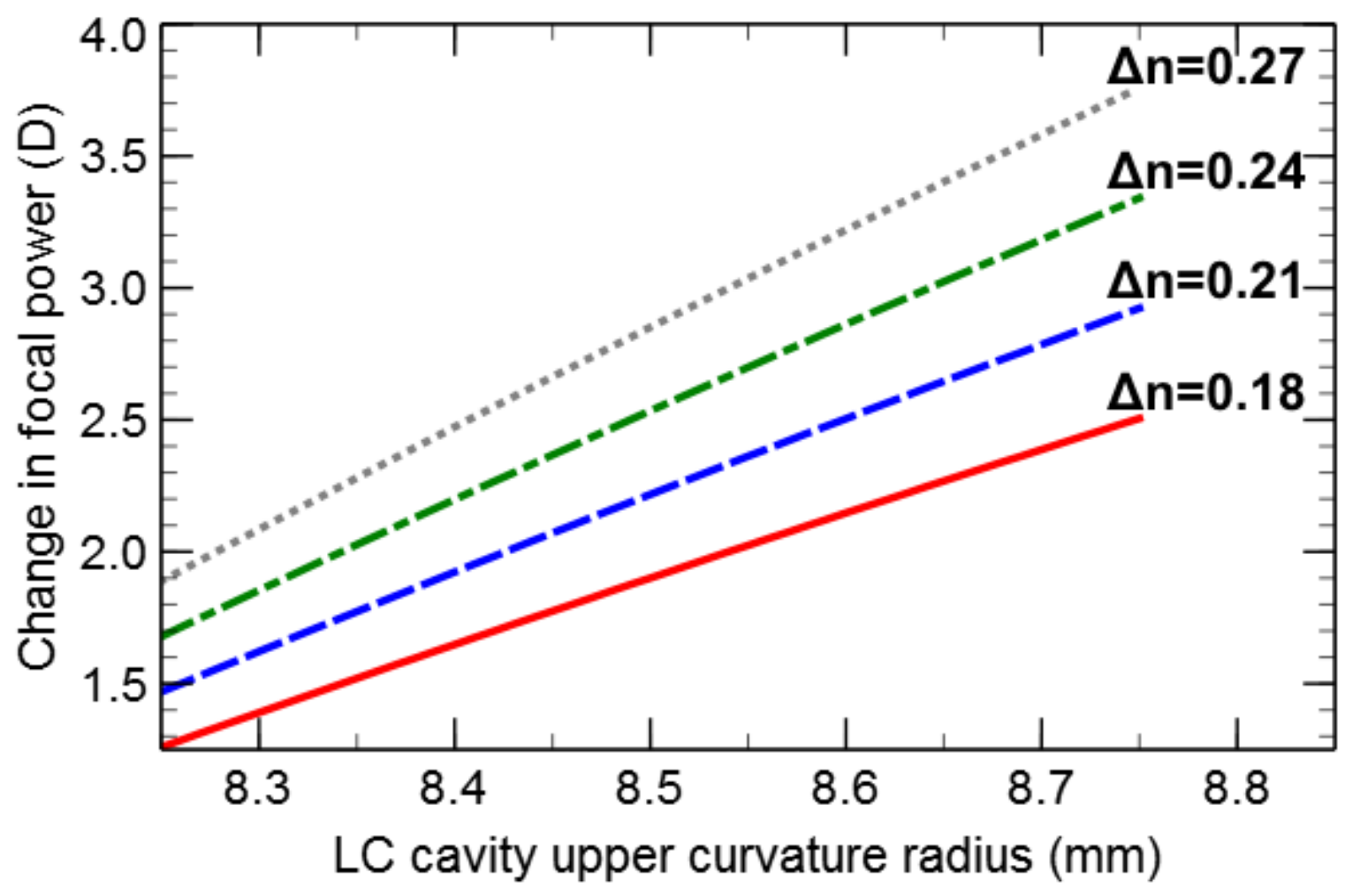

Figure 11: The greatest change in focal power with respect to the curvature of the LC cavity was calculated using equation 1 . The inner curvature of the LC cavity $r_{1}$, was kept constant, while the outer curvature $r_{2}$ was varied. Greater changes in focal power are achieved when increasing the difference in curvature between $r_{1}$ and $r_{2}$. This is more pronounced when using $L C$ 's of greater $\Delta n$.

The 2D intensity output of the beam profiler was also used to assess scattering losses in the lens, as shown in figure 12. Comparison between the lenses is complicated due to the use of the slit to sample the central area only of the lenses only. Unfortunately, this technique becomes less efficient when approaching the centre of the lens for the axial alignment. Therefore, some of these unwanted polarisations will be present when measuring the sample and could be misinterpreted as scattering. Microscopic evaluation of the lenses shows that the degree of scattering was lowest in the homeotropic $\mathrm{HH}$ and $\mathrm{HA}$ lenses.

Response times for all four modes are shown in figure 13. Caption (a) shows a comparison for the response time when switching $\mathrm{ON}$ all 4 lenses. The PA, $\mathrm{HH}$ and $\mathrm{HA}$ all switched below $1 \mathrm{~s}$ within the $2.2 \pm 0.1 \mathrm{~mm}$ diameter region tested. Furthermore, increasing the voltage also makes the lenses more responsive, with these three lenses approaching $\left\langle 0.5 \mathrm{~s}\right.$ at $>6 \mathrm{~V}_{\text {rms. }}$. The $\mathrm{PH}$ alignment is considerably slower than the other three alignment modes when switching the lens on. Large domains were observed when switching this lens, taking a few seconds for the homogenous texture to be achieved. This optical instability effectively increases the switching time of the device and causes distractive scattering to occur during the switching process. However, changing the alignment mode from $\mathrm{PH}$ to PA dramatically changed the response time, making it comparable to $\mathrm{HH}$ and HA lenses. The response times when switching the lenses OFF with respect to voltage are 

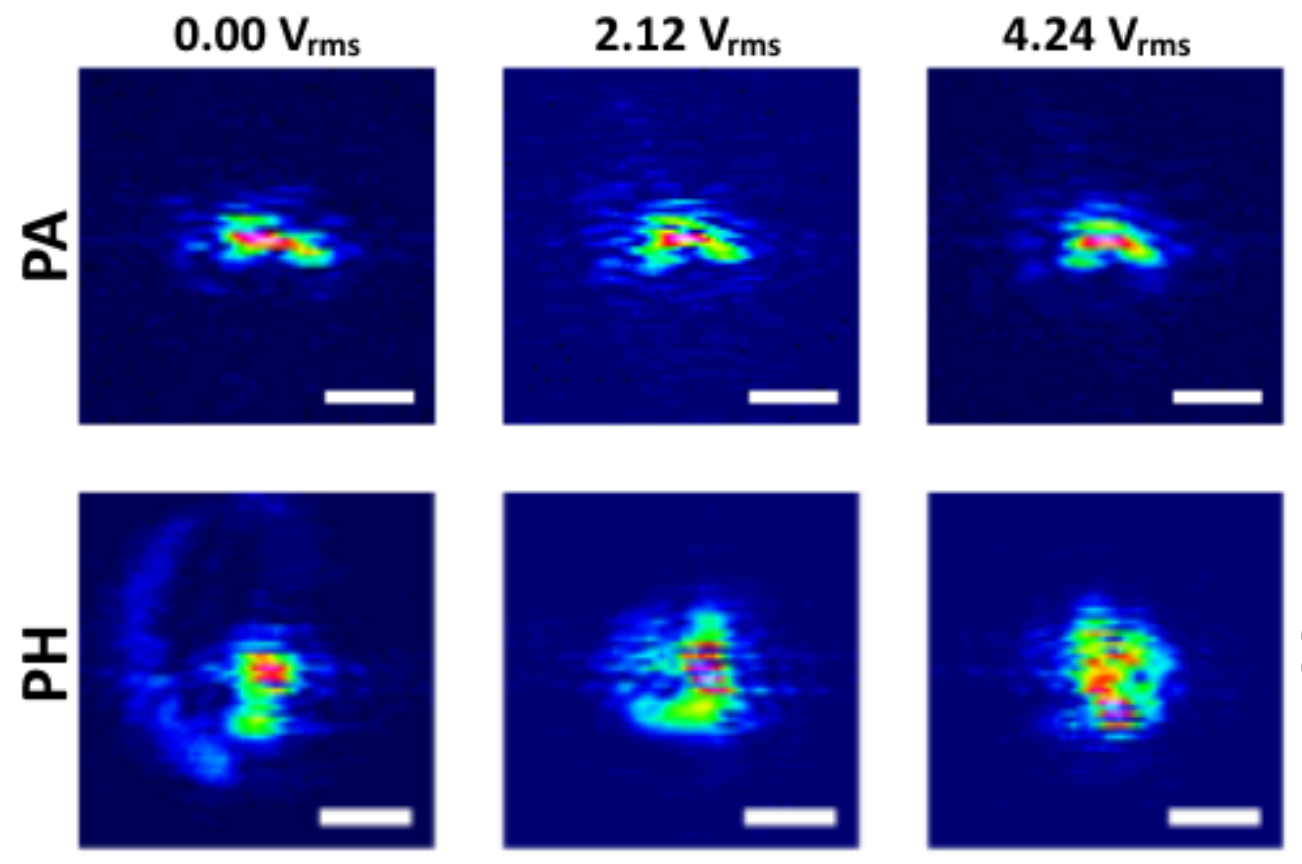

\section{$100 \%$}
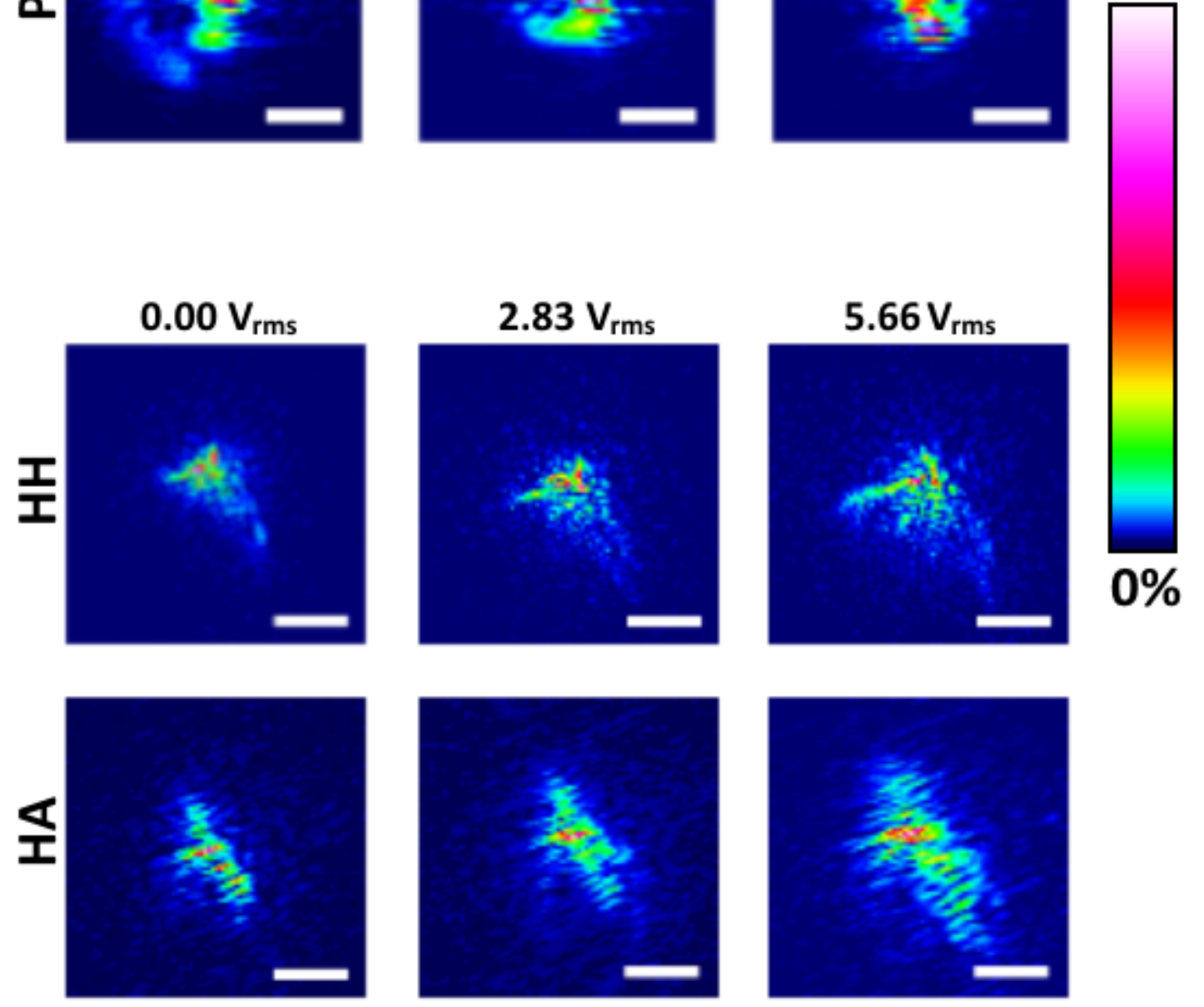

Figure 12: 2D projection of beam intensity measured using a BC106-VIS CCD beam profiler. All of the intensities are normalised so the maximum and minimum are always scalled to be $0 \%$ and $100 \%$ respectively. The axial alignment appeared to show more scattering than the homogeneous alignment for both the planar and homeotropic lenses. However, this is most likely due to the measuring technique where a slit was used instead of a radial polariser. All scale bars in this figure correspond to $250 \mu \mathrm{m}$. 
shown in caption (b). The $\mathrm{HH}, \mathrm{PH}$ and $\mathrm{PA}$ alignment modes all had comparable response times. Comparatively, the HA alignment mode has a much slower OFF response time. This is not due to error of the lens spacing, since the $\mathrm{ON}$ time was comparable to the $\mathrm{HH}$ lens. There was negligible scattering in this geometry and the cause of the slow Off HA remains unclear.

5

\section{Discussion}

The PMMA substrates used in these experiments were used due to their historical context and being more rigid and easier to use than a modern hydrogel material. However, considerations have already been made for when the PMMA substrate might be replace with a more comfortable and flexible material (such as a hydrogel). PEDOT:PSS was shown to be functional as an electrode layer for all four of the alignment modes tested. Another benefit of PEDOT:PSS was its ease of deposition, as it did not require high vacuum sputtering techniques like ITO. Inkjet printing PEDOT:PSS is also possible, which increases deposition control and reduces waste. Furthermore, polymer electronics, antenna and power supply can also be deposited using ink-jet printing, which could simplify manufacturing further.

Four different alignment techniques were tested whilst using PEDOT:PSS as an electrode layer. Both the HP and HA alignments were prepared by rubbing directly into the PEDOT:PSS, which was shown to have excellent alignment properties. HP alignment was investigated as a comparison to previous LC lens experiments $[6,8]$. Axial alignment was investigated in order to try and simplify the manufacturing process. However, this lens was more difficult to measure due to its more complex geometry. It might be possible to overcome this challenge by using either a radial polariser, or a second LC layer to make the lens polarisation independent. Homeotropic alignments were successfully achieved by depositing SE-1211 polyimide onto of the PEDOT:PSS layer. HH and HA alignments were achieved by rubbing the lenses in a planar and axial direction, respectively.

All of the lenses showed a controllable change in focal power when applying an adequate potential over the lens. This demonstrated that all alignment types can potentially be used in future LC lens applications. The PA, HH and HA alignment modes were easier to assemble than the PH lens. Domains had occurred in both of the homogeneous lenses (PH and PA), but not the homeotropic lenses $(\mathrm{HH}$ and $\mathrm{HA}$ ), as shown in figure 9. However, using axial alignment did reduce the size of the domain in the PA lens, when compared to the PH lens. This is due to the pre-tilt of the alignment layer having the same axial symmetry as the curvature of the lens, rather than being with the change in angle between the substrates for one half of the lens and against it for the other. Therefore, the difference in the pre-tilt angle between the anti-parallel alignment layers on the upper and lower surfaces is constant. This is not the case for PH alignment, as the difference between the relative pre-tilt angles for the upper and lower surfaces shifts as the director passes over the curvature of the lens. These changes result in differences of splay throughout the lens curvature, which can result in reverse tilt domains forming during the switching event. The optical quality of these lenses will diminish as these domains will cause scattering. Domains are not formed when using $\mathrm{HH}$ or HA alignment modes. 


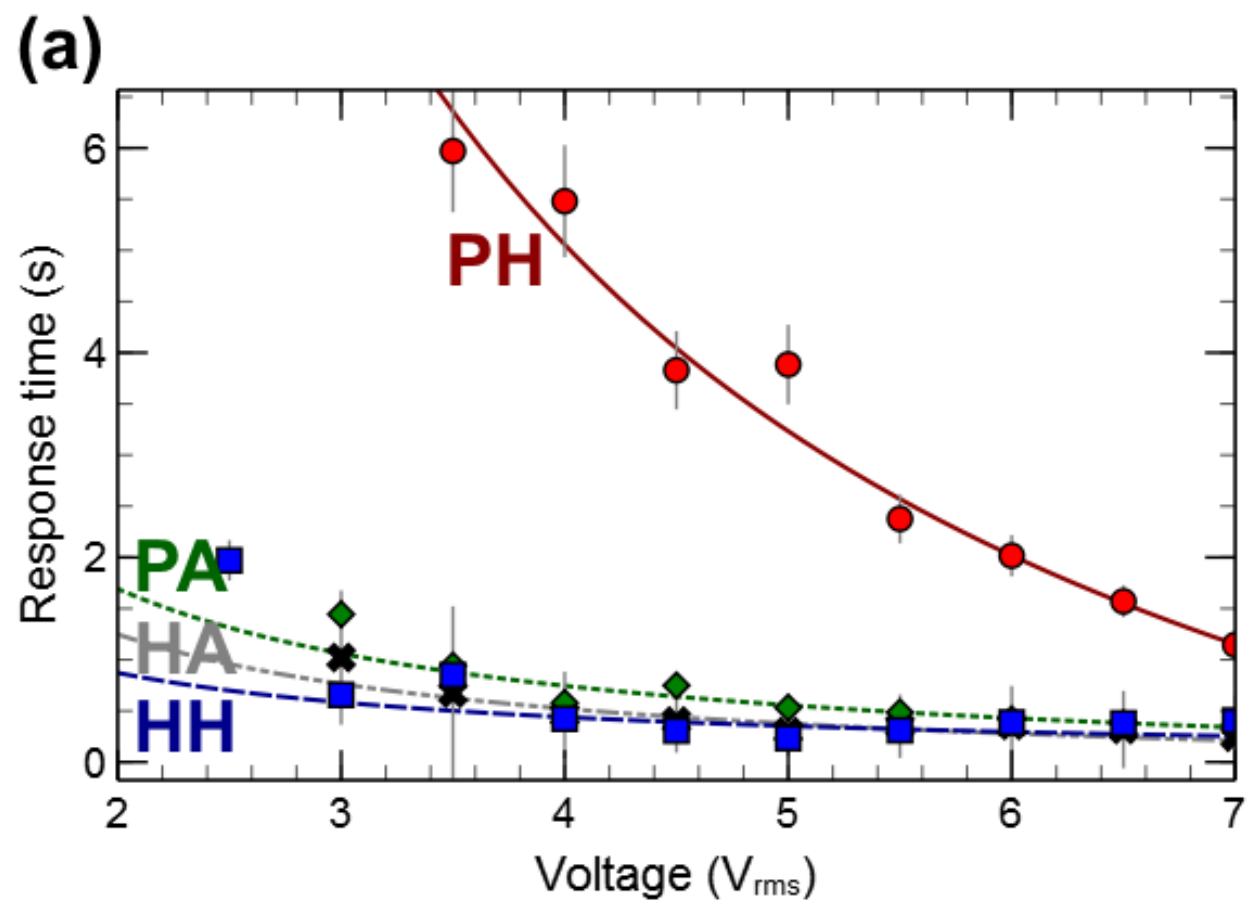

(b)

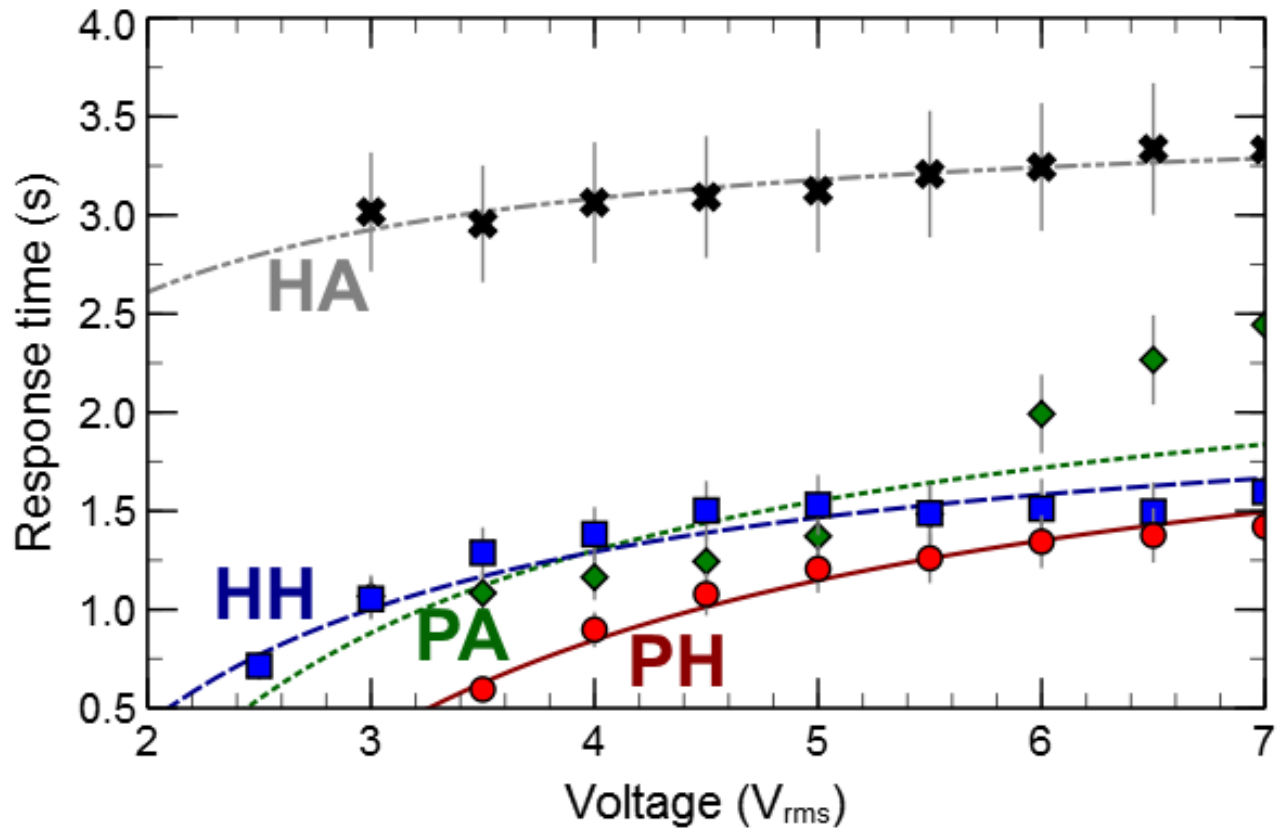

Figure 13: Lens response times for when the lenses are been switched $O N$ and OFF are shown in captions (a) and (b) respectively. The $P H, P A, H H, H A$ alignment modes were tested and shown in both plots as red circles, blue squares, green diamonds and block crosses respectively. These measurements were taken using a photo-diode, although the subjective experience of such negative lenses is likely to be much faster than the times shown here in practice. 
The overall reduction of the change in focal power between the homogeneous lenses $(\mathrm{HH}$ and $\mathrm{HA}$ ), when compared to the planar lenses (PH and PA), is due to a combination of the MLC-2081 having a lower birefringence than E7 and the difference in curvature $\left(r_{2}-r_{1}\right)$ of the LC cavity being reduced. This can be compensated using an LC with a higher $\Delta \mathrm{n}$ and/or increasing the difference in curvature between $r_{1}$ and $r_{2}$ (which would also increase $\Delta \mathrm{Y}$ and the switching times, as discussed previously). There was a greater change in focal power for the HA lens than the HH lens. This is comparable to when the change in focal power for PH was greater than PA. This could indicate that axial alignment results in greater change in focal power than homogeneous alignment. However, a larger sample set would be needed to determine whether this is an adequate conclusion, as other factors (such as small changes in lens shape) could be adding variation to these results.

Response times of the LC lenses are difficult to quantify as we do not know whether our measurements will correspond to perceived switch times by a test subject. A wearer would be more sensitive to changes at the centre of the lens. The lenses tested have a negative meniscus with the lowest spacing at the centre of the vision so that the $L C$ switches from the central region outward. Therefore, the perceived switching times is much faster than the optical measurements reported here. For this reason, a negative meniscus lens is preferable when using this type of switchable contact lens.

Figure 14 shows the lensing capability of the HH lens when ON (4.95V) and OFF (OV). A 1951 USAF resolution chart is in focus when the lens is switched OFF (near vision mode) and out of focus when switched ON (distance vision mode). Region 0 from the USAF resolution chart is shown in figure 14, where the bars and numbers can be distinguished. Switching on the lens resulted in the images coming out of focus, making them more difficult to distinguish. It was not possible to take similar images using the axial alternatives, as the two focal points from both $n_{e}$ and $n_{o}$ refractive indices images occurred when the lenses were switched ON. Using the slit would significantly diminish the field of view and a radial polariser was unavailable. Field of view is currently difficult to determine as it is usually measured subjectively by a user. The contact lenses in this paper have not yet been placed on a test subject's eye. However, the geometry is similar to that of conventional contact lenses and so a similar field of view is expected in practice.

For a nematic liquid crystal, both the refractive indices $n_{e}$ and $n_{0}$ are experienced by the incident light when the LC is orientated parallel to the surface, whereas only the $\mathrm{n}_{0}$ refractive index is observed when the $L C$ is orientated perpendicular to the surface. The $n_{0}$ refractive index must be removed when parallel as it would be disorientating to view two focal points simultaneously; this is the current problem with bifocal contact lens designs that using the liquid crystal endeavours to solve. A polarisation independent lens can be made using a short pitch, positive $\Delta \varepsilon$ cholesteric in the Grandjean texture [11], and switching to the homeotropic texture. This provides a single cavity with a phase modulation from $1 / 2\left(n_{e}+n_{0}\right)$ to $n_{0}$, which is independent of the incident polarisation. However, switching the cholesteric requires significantly higher switching voltages even for a thin device, typically $>5 \mathrm{~V} / \mu \mathrm{m}$. Such high voltages are unsuitable for use in a contact lens, where power and electronics need to be minimised. For a contact lens based on a variable spaced cavity, the voltages are considerably higher still. Alternatively, polarisation independence can be achieved using two orthogonal LC cavities in series within the lens. Each of the alignment modes previously discussed can be adapted to make a duel layer/polarisation independent LC lens. Two separate planar rubbed alignment layers will result in a polarisation independent lens if the directors of the 
two LC layers are orthogonal to one another. Likewise, this can also be achieved by applying pre-tilt to homeotropic alignment, so the directors of the two LC layers are orthogonal to one another when an electric field is applied. Axial alignment, however, requires a radially aligned counterpart layer for polarisation independence. This can be produced by radial rubbing [45], photo alignment [46], or using an orthogonal alignment layer, such as poly-N-vinylcarbazole PVK [47].

Wireless communication is necessary if the LC contact lenses are to be commercialised, so the switching of the lens can be controlled. The antenna must be fully capable of wireless communication of approximately 1 meter, relatively flexible and cheap to manufacture. A circular antenna arrangement, which avoids the pupil of the lens, is the preferred choice to prevent disruption to the lens optics. Antennas could be deposited by evaporating metallic layers onto the lens substrate. However, the majority of the lens would need to be masked and metal that is evaporated onto these areas is wasted. Inkjet printing is an efficient alternative method, as material is only deposited where it is required. There have already been previous investigations into inkjet printing metallic nano-particle suspensions to make antenna, with a sheet resistance of $380 \Omega / \square$ $[48,49]$. The focus of that research had been to make antenna for small RFID devices, which indicates that it could be readily applied to LC contact lenses.

\section{Off}

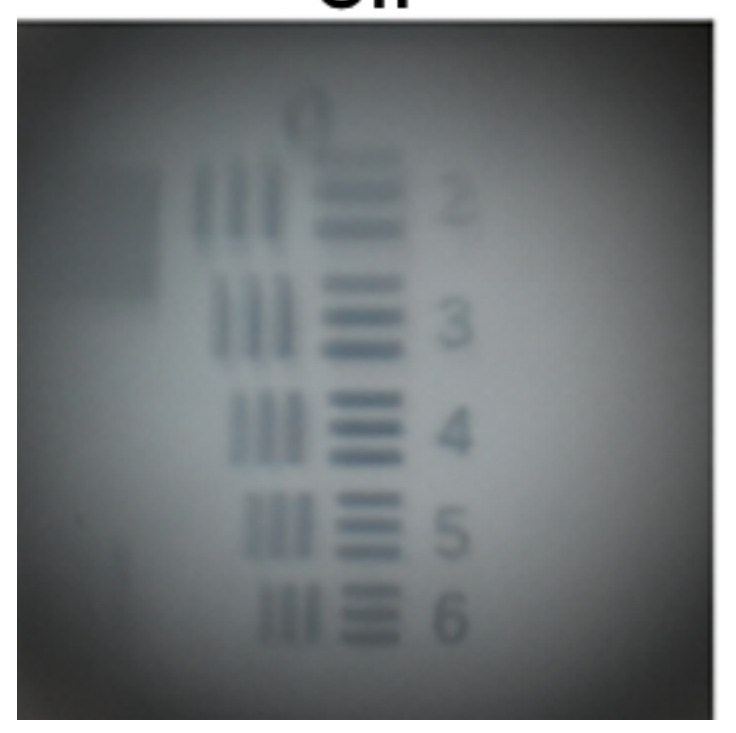

On

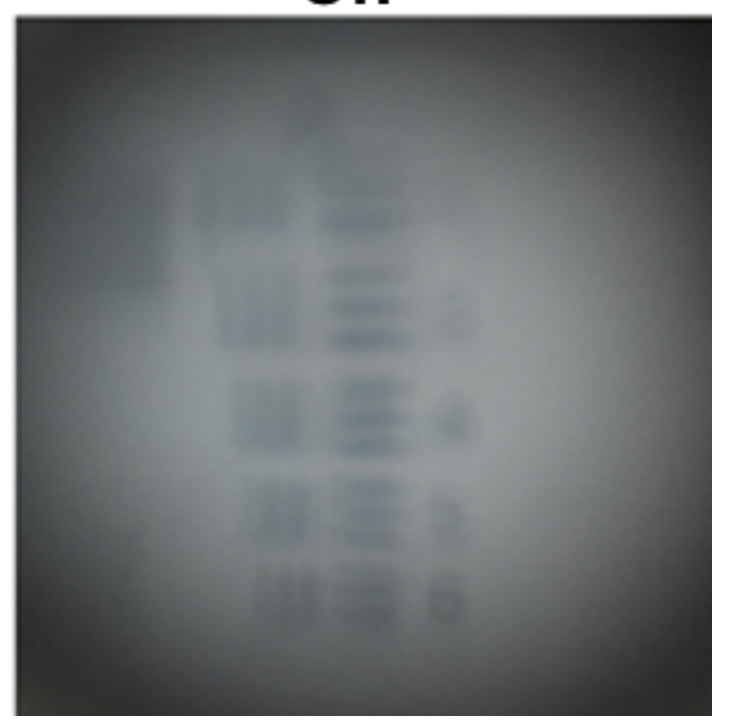

Figure 14: Demonstration of the homeotropic homogeneous contact lens switching its focus from near (off at OV) and far (on at 4.95V) distance vision modes. The test image is zone 0 of a 1951 USAF resolution chart positioned $150 \pm 5 \mathrm{~mm}$ away from the contact lens. Image was captured using a Nikon DSLR camera with its focus kept constant.

A commercialised LC lens would require its own power source so an electric field can be applied over the LC layer in its 'on state'. Like the antenna, the battery must be flexible and cheap to manufacture. It must also be located outside of the lensing optics area of the device. Polymer batteries have previously been investigated as a way to power electronic paper [50,51]. Metallic or carbon nanotubes are used as electrodes and Lithium compounds are used for the battery material. 
Both materials have been tested with screen printing, but inkjet printing could ideally be used to reduce waste. Alternatively, miniature super-capacitors could be used to power the LC contact lens, by inkjet printing electrodes either side of a dielectric material. For example, L. T. Le et. al. successfully showed that graphene oxides could be printed on kapton substrate to make an in-plane micro-capacitor [52]. This could be adapted by ink-jet printing the capacitor onto a LC lens. Therefore, most of the electronics can be inkjet-printed, which simplifies manufacturing and lowers costs.

\section{Conclusion:}

Different manufacturing and alignment techniques have been investigated in order to develop LC contact lenses towards a functioning product. The electrodes, alignment modes and LC lens cavity shape have all been considered and tested. However, other components of the lenses still need to be investigated before a fully functioning device can be commercially prepared. These include the antenna, power supply and electronics. We propose that most of these components can be prepared/ deposited directly onto the lens using inkjet printing. Therefore, most of the lens would be prepared using ink-jet printing techniques, where the lens could be prepared using a single system.

The POM images shown in figure 9 clearly showed that there are differences between all of the alignment types tested. Our optical measurements indicated that larger changes in focal power are possible by using axial geometries (PA and HA). However, simple calculations demonstrated that small changes in the lens shape could dramatically increase/decrease the change in focal power from these devices. Only 4 lenses were prepared and tested and a larger sample set would be needed to determine the impact of experimental variation.

There were no domains in the $\mathrm{HH}$ and $\mathrm{HA}$ aligned lenses, unlike the $\mathrm{PH}$ and PA devices. However, the HA lens demonstrated a greater change in focal power, which could be due to small changes in the lens shape changing the optical properties of the device (as previously discussed). These results should be complimented in future by thorough modelling of the LC contact lens to determine if there is any benefit for using $\mathrm{HA}$ over $\mathrm{HH}$. Refining the choices towards the optimum alignment mode is critical towards commercialising the liquid crystal contact lenses. We have reported the experimental results for 4 of these modes and concluded that modelling should be done for a deeper understanding of their effects.

\section{Acknowledgement:}

The authors wish to than the EPSRC for funding this work under an Advanced Manufacturing Fellowship for JCJ (EP/L015188/1). 


\section{References:}

[1] Charman W N 2005 Restoring accommodation: A dream or an approaching reality? Ophthal. Physiol. Opt. 25 1-6

[2] Morgan P B and Efron N 2009 Contact lens correction of presbyopia Cont. Lens Anterior Eye 32 191-2

[3] Fricke T R, Holden B A, Wilson D A, Schlenther G, Naidoo K S, Resnikoff S and Frick K D 2012 Global cost of correcting vision impairment from uncorrected refractive error Bull World Heal. Organ 90 728-38

[4] Goertz A D, Stewart W C, Burns W R, Stewart J A and Nelson L A 2014 Review of the impact of presbyopia on quality of life in the developing and developed world Acta Ophthalmol. 92 497-500

[5] Luo B P, Brown G C, Luo S C and Brown M M 2008 The Quality of Life Associated with Presbyopia Am. J. Ophthalmol. 145 618-22

[6] Milton H E, Morgan P B, Clamp J H and Gleeson H F 2014 Electronic liquid crystal lenses for the correction of presbyopia Opt. Express 22 8035-40

[7] Milton H E, Gleeson H F, Morgan P B, Goodby J W, Cowling S and Clamp J H 2014 Switchable liquid crystal contact lenses: dynamic vision for the ageing eye Proc. of SPIE vol 9004, ed L-C Chien, A M Figueiredo Neto, K Neyts and M Ozaki p 90040H

[8] Syed I M, Kaur S, Milton H E, Mistry D, Bailey J, Morgan P B, Jones J C and Gleeson H F 2015 Novel switching mode in a vertically aligned liquid crystal contact lens Opt. Express 23 9911-6

[9] Sato S 1979 Liquid-Crystal Lens-Cells With Variable Focal Length Jpn. J. Appl. Phys. 18 167984

[10] Sato S, Suguyama A and Sato R 1985 Variable-Focus Liquid-Crystal Fresnel Lens Jpn. J. Appl. Phys. 24 L626-8

[11] Large T A 1994 Switchable Lens, Patent \#: US 00571272 (1A) 7

[12] Ye M, Wang B and Sato S 2004 Liquid-crystal lens with a focal length that is variable in a wide range. Appl. Opt. 43 6407-12

[13] Naumov A F, Love G D, Loktev M Y and Vladimirov F L 1999 Control optimization of spherical modal liquid crystal lenses. Opt. Express 4 344-52

[14] Li G, Mathine D L, Valley P, Ayräs P, Haddock J N, Giridhar M S, Williby G, Schwiegerling J, Meredith G R, Kippelen B, Honkanen S and Peyghambarian N 2006 Switchable electro-optic diffractive lens with high efficiency for ophthalmic applications. Proc. Natl. Acad. Sci. U. S. A. $1036100-4$

[15] Fujita T, Nishihara $\mathrm{H}$ and Koyama J 1981 Fabrication of micro lenses using electron-beam lithography. Opt. Lett. 6 613-5

[16] Ren H and Wu S-T 2006 Adaptive liquid crystal lens with large focal length tunability. Opt. Express 14 11292-8

[17] Ren H, Fox D W, Wu B and Wu S-T 2007 Liquid crystal lens with large focal length tunability and low operating voltage Opt. Express 1511328 
[18] Lin Y-H, Ren H, Wu Y-H, Zhao Y, Fang J, Ge Z and Wu S-T 2005 Polarization-independent liquid crystal phase modulator using a thin polymer-separated double-layered structure. Opt. Express 13 8746-52

[19] Ren H, Fan Y H, Gauza S and Wu S T 2004 Tunable-focus flat liquid crystal spherical lens Appl. Phys. Lett. 84 4789-91

[20] Kaur S, Kim Y-J, Milton H, Mistry D, Syed I M, Bailey J, Novoselov K S, Jones J C, Morgan P B, Clamp J and Gleeson H F 2016 Graphene electrodes for adaptive liquid crystal contact lenses Opt. Express 24 8782-7

[21] Forrester J V., Dick A D, McMenamin P G and Roberts F 2007 The Eye: Basic Sciences in Practice (Philadelphia, United States: Saunders LTD)

[22] Goodby J W, Collings P J, Kato T, Tschierske C, Gleeson H F and Raynes P 2014 Handbook of Liquid Crystals (Weinheim, Germany: Wiley-VCH)

[23] Kumar A and Zhou C 2010 The race to replace tin-doped indium oxide: Which material will win? ACS Nano 4 11-4

[24] Vasant Kumar C V R and Mansingh A 1989 Effect of target-substrate distance on the growth and properties of rf-sputtered indium tin oxide films J. Appl. Phys. 65 1270-80

[25] Kim H, Horwitz J S, Kushto G, Piqué A, Kafafi Z H, Gilmore C M and Chrisey D B 2000 Effect of film thickness on the properties of indium tin oxide thin films J. Appl. Phys. 88 6021-5

[26] Cairns D R, Witte II R P, Sparacin D K, Sachsman S M, Paine D C, Crawford G P and Newton R $R 2000$ Strain-dependent electrical resistance of tin-doped indium oxide on polymer substrates Appl. Phys. Lett. 76 1425-7

[27] Park S K, Han J I, Moon D G and Kim W K 2003 Mechanical stability of externally deformed indium-tin-oxide films on polymer substrates Japanese J. Appl. Physics, Part 1 Regul. Pap. Short Notes Rev. Pap. 42 623-9

[28] Cairns D R, Gorkhali S P, Esmailzadeh S, Vedrine J and Crawford G P 2003 Conformable displays based on polymer-dispersed liquid-crystal materials on flexible substrates J. Soc. Inf. Disp. 11 289-95

[29] Hohnholz D, Okuzaki H and MacDiarmid A G 2005 Plastic electronic devices through line patterning of conducting polymers Adv. Funct. Mater. 15 51-6

[30] Langley D, Giusti G, Mayousse C, Celle C, Bellet D and Simonato J-P 2013 Flexible transparent conductive materials based on silver nanowire networks: a review. Nanotechnology 24 452001

[31] Lee E H, Ryu J H, Jang J and Park K C 2011 Patterned Single-Wall Carbon Nanotube Transparent Conducting Films for Liquid Crystal Switching Electrodes Jpn. J. Appl. Phys. 5003CA04

[32] Chan Yu King R and Roussel F 2007 Transparent carbon nanotube-based driving electrodes for liquid crystal dispersion display devices Appl. Phys. A Mater. Sci. Process. 86 159-63

[33] Blake P, Brimicombe P D, Nair R R, Booth T J, Jiang D, Schedin F, Ponomarenko L a., Morozov S V., Gleeson H F, Hill E W, Geim A K and Novoselov K S 2008 Graphene-based liquid crystal device. Nano Lett. 8 1704-8 
[34] Kostianovskii V, Sanyoto B and Noh Y Y 2017 A facile way to pattern PEDOT:PSS film as an electrode for organic devices Org. Electron. physics, Mater. Appl. 44 99-105

[35] Singh A, Katiyar M and Garg A 2015 Understanding the formation of PEDOT:PSS films by inkjet printing for organic solar cell applications RSC Adv. 5 78677-85

[36] Shi Y, Liu J and Yang Y 2000 Device performance and polymer morphology in polymer light emitting diodes: The control of thin film morphology and device quantum efficiency J. Appl. Phys. 87 4254-63

[37] Padinger F, Rittberger R S and Sariciftci N S 2003 Effects of postproduction treatment on plastic solar cells Adv. Funct. Mater. 13 85-8

[38] Fraval $\mathrm{N}$ and de Bougrenet de la Tocnaye J L 2010 Low aberrations symmetrical adaptive modal liquid crystal lens with short focal lengths. Appl. Opt. 49 2778-83

[39] Son S, Pugal D, Hwang T, Choi H R, Koo J C, Lee Y, Kim K and Nam J-D 2012 Electromechanically driven variable-focus lens based on transparent dielectric elastomer Appl. Opt. 51 2987-96

[40] Chen H-S, Wang Y-J, Chang C-M and Lin Y-H 2015 A Polarizer-Free Liquid Crystal Lens Exploiting an Embedded-Multilayered Structure Photon. Technol. Lett. 27 899-902

[41] De Smet J, Avci A, Joshi P, Cuypers D and De Smet H 2012 A Liquid Crystal Based Contact Lens Display Using PEDOT : PSS and Obliquely Evaporated SiO2 SID 2012 pp 1375-8

[42] De Smet J, Avci A, Beernaert R, Cuypers D and De Smet H 2012 Design and Wrinkling Behavior of a Contact Lens With an Integrated Liquid Crystal Light Modulator J. Disp. Technol. 8 299-305

[43] De Smet J, Avci A, Joshi P, Schaubroeck D, Cuypers D and De Smet H 2014 Progress toward a liquid crystal contact lens display J. Soc. Inf. Disp. 21 399-406

[44] Hecht E 2008 Optics (Boston, United States: Addison Wesley)

[45] Vela C C, Quintana X, Otón E, Geday M A and Otón J M 2011 Security devices based on liquid crystals doped with a colour dye Opto-Electron. Rev. 19 496-500

[46] Huang C Y, Tsai H Y, Wang Y H, Huang C M, Lo K Y and Lee C R 2010 Linear polarization rotators based on dye-doped liquid crystal cells Appl. Phys. Lett. 96 1-3

[47] Chen Y, Ying-guey Fuh A, Liu C and Cheng K-T 2011 Radial liquid crystal alignment based on circular rubbing of a substrate coated with poly ( $\mathrm{N}$-vinyl carbazole ) film J. Phys. D Appl. Phys. 44 1-5

[48] Yang L, Rida A, Vyas R and Tentzeris M M 2007 RFID tag and RF structures on a paper substrate using inkjet-printing technology IEEE Trans. Microw. Theory Tech. 55 2894-901

[49] Rida A, Yang L, Vyas R and Tentzeris M M 2009 Conductive inkjet-printed antennas on flexible low-cost paper-based substrates for RFID and WSN applications IEEE Antennas Propag. Mag. 51 13-23

[50] Hu L, Wu H, La Mantia F, Yang Y and Cui Y 2010 Thin, flexible secondary Li-ion paper batteries ACS Nano 4 5843-8

[51] Agrawal R C and Pandey G P 2008 Solid polymer electrolytes: materials designing and all- 
solid-state battery applications: an overview J. Phys. D. Appl. Phys. 41223001

[52] Le L T, Ervin M H, Qiu H, Fuchs B E, Zunino J and Lee W Y 2011 Inkjet-printed graphene for flexible micro-supercapacitors Proc. IEEE Conf. Nanotechnol. 67-71. 